\title{
Proper-motion binaries in the Hipparcos catalogue
}

\section{Comparison with radial velocity data ${ }^{\star}$}

\author{
A. Frankowski, S. Jancart, and A. Jorissen ${ }^{\star \star}$ \\ Institut d'Astronomie et d'Astrophysique, Université Libre de Bruxelles, CP 226, Boulevard du Triomphe, 1050 Bruxelles, Belgium \\ Received 30 April 2006 / Accepted 16 October 2006 \\ ABSTRACT

\begin{abstract}
Context. This paper is the last in a series devoted to the analysis of the binary content of the Hipparcos Catalogue.
Aims. The comparison of the proper motions constructed from positions spanning a short (Hipparcos) or long time (Tycho-2) makes it possible to uncover binaries with periods of the order of or somewhat larger than the short time span (in this case, the $3 \mathrm{yr}$ duration of the Hipparcos mission), since the unrecognised orbital motion will then add to the proper motion.

Methods. A list of candidate proper motion binaries is constructed from a carefully designed $\chi^{2}$ test evaluating the statistical significance of the difference between the Tycho-2 and Hipparcos proper motions for 103134 stars in common between the two catalogues (excluding components of visual systems). Since similar lists of proper-motion binaries have already been constructed, the present paper focuses on the evaluation of the detection efficiency of proper-motion binaries, using different kinds of control data (mostly radial velocities). The detection rate for entries from the Ninth Catalogue of Spectroscopic Binary Orbits $\left(S_{\mathrm{B}^{9}}\right)$ is evaluated, as well data in the Geneva-Copenhagen survey of F and $\mathrm{G}$ dwarfs in the solar neighbourhood.

Results. Proper motion binaries are efficiently detected for systems with parallaxes in excess of $\sim 20$ mas, and periods in the range 1000-30 $000 \mathrm{~d}$. The shortest periods in this range (1000-2000 d, i.e., once to twice the duration of the Hipparcos mission) may appear only as DMSA/G binaries (accelerated proper motion in the Hipparcos Double and Multiple System Annex). Proper motion binaries detected among $S_{\mathrm{B}^{9}}$ systems having periods shorter than about $400 \mathrm{~d}$ hint at triple systems, the proper-motion binary involving a component with a longer orbital period. A list of 19 candidate triple systems is provided. Binaries suspected of having low-mass dence for duplicity whatsoever (be it spectroscopic or astrometric). Finally, the fraction of proper-motion binaries shows no significant
\end{abstract} \\ as for stars like barium stars, which are known to be all binaries, and finally for spectroscopic binaries identified from radial velocity \\ (brown-dwarf-like) companions are listed as well. Among the 37 barium stars with parallaxes larger than 5 mas, only 7 exhibit no evi- \\ variation among the various (regular) spectral classes, when due account is taken for the detection biases.
}

Key words. astrometry - binaries: general - catalogs - binaries: spectroscopic - binaries: visual

\section{Introduction}

Among the 118218 entries in the Hipparcos Catalogue (ESA 1997), about 18000 (collected in the Double and Multiple Systems Annex - DMSA) involve binary or multiple stars, which break down as follows (Sect. 1.04 of ESA 1997): 13211 entries are component solutions (i.e., visual double or multiple systems, DMSA/C), 2622 are acceleration solutions (i.e., with a variable proper motion, or DMSA/G), 235 astrometric binaries have a known orbit (DMSA/O), 288 are VariabilityInduced Movers (VIMs, or DMSA/V) and finally 1561 are stochastic solutions (DMSA/X), a number of which might be binaries. With the exception of component solutions, all the other kinds of binary entries have been re-evaluated in the present series of papers (Pourbaix et al. 2003; Platais et al. 2003; Pourbaix 2004; Jorissen et al. 2004; Jancart et al. 2005). Among the 235 DMSA/O systems, 122 have been re-investigated by Jancart et al. (2005) in light of their spectroscopic orbital elements, and 8 orbital solutions were rejected because of their low quality (Sect. 4.3 of Jancart et al. 2005). At the same time, 51 new

* Full Table 2 is only available in electronic form at the CDS via anonymous ftp to cdsarc.u-strasbg.fr (130.79.128.5) or via http://cdsweb.u-strasbg.fr/cgi-bin/qcat?]/A+A/464/377

$\star \star$ Senior Research Associate, F.N.R.S., Belgium.
DMSA/O were found using their spectroscopic elements published after the completion of the Hipparcos Catalogue, and collected in the Ninth Catalogue of Spectroscopic Binary Orbits $\left(S_{\mathrm{B}^{9}}\right.$; Pourbaix et al. 2004). For the 188 DMSA/V entries that are long-period variables, the re-processing of their Hipparcos data (using a variable chromaticity correction) led to the rejection of 161 of them (Pourbaix et al. 2003).

Even when spectroscopic orbital elements are not available, for each star from the Hipparcos Catalogue (with the exception of the DMSA/C component solutions), it is possible to quantify the likelihood that their Intermediate Astrometric Data (IAD; van Leeuwen \& Evans 1998) are better fitted with an orbital model than with a standard single-star model (i.e., a 5-parameter model, including the parallax $\varpi$, the $\alpha$ and $\delta$ positions at the 1991.25 reference epoch, and the proper motion in $\alpha$ and $\delta$ ). Hipparcos data are, however, seldom precise enough to derive the orbital elements from scratch. Therefore, when a spectroscopic orbit is available beforehand, it is advantageous to import $e, P, T$ from the spectroscopic orbit and to derive the remaining astrometric elements (as done by Pourbaix \& Jorissen 2000; Pourbaix \& Boffin 2003; Jancart et al. 2005). If a spectroscopic orbit is not available, trial $(e, P, T)$ triplets scanning a regular grid (with $10 \leq P(\mathrm{~d}) \leq 5000$ imposed by the Hipparcos scanning law and the mission duration) may be used. In practice, it is even possible to avoid scanning $e$ and $T$, and to restrict the grid to the orbital period $P$, assuming circular orbits. This 
choice greatly reduces the computing time, since test cases revealed that binary systems with moderate eccentricities are nevertheless detected even though they are fitted with circular orbits (see Pourbaix 2004, for details). Very eccentric orbits might be missed, of course, but their small number does not justify the tremendous increase in computer time required to detect them. This method has been applied to barium stars by Jorissen et al. $(2004,2005)$, and has been shown to provide the best detection efficiency if $\varpi>5$ mas and $100 \leq P(\mathrm{~d}) \leq 4000$.

The present paper is the last one in our series reprocessing binaries in the Hipparcos Catalogue. It deals with binaries that are identifiable on the ground of their variable proper motion. The DMSA already contains 2622 such DMSA/G solutions. This paper aims at extending the list of proper-motion binaries $^{1}$ by comparing the Hipparcos proper motion with the Tycho-2 proper motion (Høg et al. 2000a,b), following the idea put forward by Wielen (1997) and Wielen et al. (1998, 1999). Kaplan \& Makarov (2003), Makarov (2004), and Makarov \& Kaplan (2005) presented further developments and applications. The Hipparcos proper motion, being based on observations spanning only $3 \mathrm{yr}$, may be altered by the orbital motion, especially for systems with periods in the range 1500 to $30000 \mathrm{~d}$ whose orbital motion was not recognised by Hipparcos. On the contrary, this effect should average out in the Tycho-2 proper motion, which is derived from observations covering a much longer time span. This method works best when applied to stars with parallaxes in excess of about 20 mas. The method is fully described in Sect. 2.

While the present paper was being prepared, Makarov \& Kaplan (2005) issued the list of proper-motion binaries at which we were aiming. Wielen et al. (1999) published a list of proper-motion binaries (collected in the DMUBIN database: http://www.ari.uni-heidelberg.de/ datenbanken/dmubin), based on the comparison of Hipparcos proper motions with several other catalogues, as well. We nevertheless believe that the present analysis remains timely, for the following reasons: (i) the statistical method used here to identify proper-motion binaries is different from the one of Makarov \& Kaplan (2005). Our method takes into account the correlation between the $\alpha$ and $\delta$ components of the Hipparcos proper motion, as well as between the Hipparcos and Tycho-2 proper motions, though on a statistical rather than individual manner for the latter. (ii) We illustrate the astrophysical promises of the method by applying it to specific classes of stars like barium stars (Sect. 5.2), Am stars, or Wolf-Rayet stars (Sect. 6). Among those classes, binaries are sometimes difficult to find using the traditional spectroscopic approach. For instance, in the case of barium stars, the periods may be quite long. (iii) In Sect. 5.1, we test the detectability criteria of proper-motion binaries devised by Makarov \& Kaplan (2005) by looking for such binaries in the Ninth Catalogue of Spectroscopic Binary Orbits ( $S_{\mathrm{B}^{9}}$; Pourbaix et al. 2004). (iv) The cross-check between the list of candidate proper-motion binaries and the corresponding radial-velocity data (available for a large sample of stars from the HIPPARCOS/CORAVEL survey; Udry et al. 1997) allows us to identify spurious proper-motion binaries (due to perspective acceleration) or binaries with low-mass (brown-dwarf-like) companions (Sect. 5.3). (v) We evaluate the proportion of propermotion binaries among the different spectral classes (Sect. 6) and do not confirm Makarov \& Kaplan's (2005) suggestion that the

\footnotetext{
1 The concept of proper-motion binaries, which is the topic of the present paper, should not be confused with the more traditional one of common proper-motion pairs.
}

fraction of long-period binaries is larger among solar-type stars than among the other spectral classes.

\section{Statistical method}

The Tycho-2 Catalogue (Høg et al. 2000a,b) is an extension of the Tycho Catalogue collected by the sky mapper of the ESA Hipparcos satellite (ESA 1997). It contains astrometric positions and proper motions for $2.5 \times 10^{6}$ stars. The proper motions were extracted from the Hipparcos and Tycho- 2 catalogues for those stars with only one component, thus excluding the DMSA/C and DMSA/O entries, as well as Hipparcos objects that have double entries in the Tycho- 2 catalogue. We ended up with 103134 stars. The difference between the Hipparcos and Tycho- 2 proper motions is then evaluated through a $\chi^{2}$ test:

$\chi^{2}=\boldsymbol{X}^{\mathrm{t}} S^{-1} \boldsymbol{X}$

where $\boldsymbol{X}$ is the two-dimensional vector containing the difference between the Hipparcos and Tycho- 2 proper motions, the first component corresponding to the right ascension $\alpha$ and the second to the declination $\delta$. The $2 \times 2$ covariance matrix $S$ on the proper-motion differences is obtained from the $4 \times 4$ covariance matrix $\Sigma$ on the proper motions themselves by the relation

$S=A \Sigma A^{\mathrm{t}}$

where $A$ is a $2 \times 4$ matrix such that

$\boldsymbol{X}=A \boldsymbol{Y} \equiv\left(\begin{array}{rrrr}1 & 0 & -1 & 0 \\ 0 & 1 & 0 & -1\end{array}\right) \boldsymbol{Y}$,

where $\boldsymbol{Y}$ is the 4-dimensional vector containing the $\alpha$ and $\delta$ components of the Hipparcos proper motion followed by the $\alpha$ and $\delta$ components of the Tycho-2 proper motion. The covariance matrix $\Sigma$ associated with the proper-motion vector $\boldsymbol{Y}$ is expressed by:

$$
\begin{aligned}
& \Sigma \equiv \\
& \left(\begin{array}{ccccc}
\sigma_{\alpha \mathrm{H}}^{2} & \rho_{\mathrm{H}} \sigma_{\alpha \mathrm{H}} \sigma_{\delta \mathrm{H}} & \rho_{\mathrm{HT}, \alpha} & \sigma_{\alpha \mathrm{H}} \sigma_{\alpha \mathrm{T}} & 0 \\
\rho_{\mathrm{H}} \sigma_{\alpha \mathrm{H}} \sigma_{\delta \mathrm{H}} & \sigma_{\delta \mathrm{H}}^{2} & 0 & \rho_{\mathrm{HT}, \delta} \sigma_{\delta \mathrm{H}} \sigma_{\delta \mathrm{T}} \\
\rho_{\mathrm{HT}, \alpha} \sigma_{\alpha \mathrm{H}} \sigma_{\alpha \mathrm{T}} & 0 & \sigma_{\alpha \mathrm{T}}^{2} & \rho_{\mathrm{T}} \sigma_{\alpha \mathrm{T}} \sigma_{\delta \mathrm{T}} \\
0 & \rho_{\mathrm{HT}, \delta} \sigma_{\delta \mathrm{H}} \sigma_{\delta \mathrm{T}} & \rho_{\mathrm{T}} \sigma_{\alpha \mathrm{T}} \sigma_{\delta \mathrm{T}} & \sigma_{\delta \mathrm{T}}^{2}
\end{array}\right) .
\end{aligned}
$$

Here $\sigma_{\alpha \mathrm{H}}$ and $\sigma_{\delta \mathrm{H}}$ are the standard errors of the Hipparcos proper-motion $\alpha$ and $\delta$ components, from fields $\mathrm{H} 17$ and $\mathrm{H} 18$ of the Hipparcos Catalogue, and $\sigma_{\alpha T}$ and $\sigma_{\delta T}$ are the respective standard errors of the Tycho-2 proper motion, from fields T17 and T18 of the Tycho- 2 Catalogue. The correlation coefficient $\rho_{\mathrm{H}}$ between the $\alpha$ and $\delta$ component of the Hipparcos proper motion is taken from field $\mathrm{H} 28$ of the Hipparcos Catalogue. The analogous correlation $\rho_{\mathrm{T}}$ for the Tycho- 2 catalogue is supposed to be null, given the different approach used to construct the Tycho-2 catalogue. The correlation coefficients $\rho_{\mathrm{HT}, \alpha}$ and $\rho_{\mathrm{HT}, \delta}$ between the Hipparcos and Tycho-2 proper motions were evaluated by Høg et al. (2000a), based on a sample of supposedly single stars. To quantify these correlations, these authors define a parameter $R$ (separately for the $\alpha$ and $\delta$ components; when no ambiguity is possible, the subscript will therefore be omitted, for the sake of simplicity) as

$$
R^{2}=\frac{\sigma_{\mathrm{d}}^{2}}{\sigma_{\mathrm{H}}^{2}+\sigma_{\mathrm{T}}^{2}+\sigma_{\mathrm{c}}^{2}}
$$

and

$\sigma_{\mathrm{d}}^{2}=\sigma_{\mathrm{H}}^{2}+\sigma_{\mathrm{T}}^{2}-2 \rho_{\mathrm{HT}} \sigma_{\mathrm{H}} \sigma_{\mathrm{T}}+\sigma_{\mathrm{c}}^{2}$, 
Table 1. The quantities $r_{\alpha}$ and $r_{\delta}$ as a function of the Tycho magnitude $V_{\mathrm{T}}$ (see Eq. (7)).

\begin{tabular}{lll}
\hline \hline$V_{\mathrm{T}}$ & $r_{\alpha}$ & $r_{\delta}$ \\
\hline$<7.0$ & 1. & 1. \\
$7.0-8$ & 0.90 & 0.95 \\
$8.0-9$ & 0.92 & 0.94 \\
$9.0-10$ & 0.89 & 0.93 \\
$10.0-11$ & 0.95 & 0.99 \\
$11.0-12$ & 1.03 & 1.09 \\
$>12.0$ & 0.98 & 1.13 \\
\hline
\end{tabular}

$\sigma_{\mathrm{c}}$ being a "cosmic error". The quantity $\sigma_{\mathrm{c}}$ in fact accounts for the presence of unrecognised astrometric binaries, which artificially increases $\sigma_{\mathrm{d}}$ when comparing proper motions from two different catalogues (Wielen 1997; Wielen et al. 1998). This is precisely the effect we are after. Therefore, including the cosmic error in the evaluation of $R$, which is itself used in the $\chi^{2}$ test (Eq. (1)), would in fact introduce a bias in the method. Therefore, a new quantity $r$ has been evaluated, closely following the definition of $R$ (Eq. (5)), but without the cosmic error term. It is listed in Table 1 and is derived from the $R$ values listed in Table 1 of $\mathrm{H} ø \mathrm{~g}$ et al. (2000a) using the following expression:

$r^{2} \equiv \frac{\sigma_{\mathrm{d}}^{2}}{\sigma_{\mathrm{H}}^{2}+\sigma_{\mathrm{T}}^{2}}=\frac{1}{\frac{1}{R^{2}}-\frac{\sigma_{\mathrm{c}}^{2}}{\sigma_{\mathrm{d}}^{2}}}$.

The parameter $R$ was obtained by Høg et al. (2000a) for bins of 1 mag from $V_{\mathrm{T}}=7.0$ to 12.0 (restricted to subsamples of nonbinary stars), by computing $\sigma_{\mathrm{d}}$ and the rms values of the internal errors $\sigma_{\mathrm{H}}$ and $\sigma_{\mathrm{T}}$ of the proper motions given in the two catalogues, plus an estimate of the cosmic error. It is important to stress at this point that no $R$ factor is available for the brightest stars $\left(V_{\mathrm{T}}<7\right)$. These bright stars were not considered in the derivation of $R$ as the vast majority of them, having accurate Hipparcos data, determine the systems of the other catalogues, from which the Tycho-2 proper motions are derived.

The parameter $r$ thus expresses the ratio between the standard deviation $\sigma_{\mathrm{d}}$ of the difference in Hipparcos and Tycho-2 proper motions and the root-mean square of the internal errors for these two catalogues. In the absence of any correlation, $r$ should be unity; in the presence of correlation, $\rho_{\mathrm{HT}}$ may be expressed

$\rho_{\mathrm{HT}} \sigma_{\mathrm{H}} \sigma_{\mathrm{T}}=\frac{1}{2}\left(1-r^{2}\right)\left(\sigma_{\mathrm{H}}^{2}+\sigma_{\mathrm{T}}^{2}\right)$.

It should be noted, however, that a value of $r$ different from unity may also indicate that the "internal errors" $\sigma_{\mathrm{H}}$ or $\sigma_{\mathrm{T}}$ for the proper motions from the Hipparcos and Tycho-2 catalogues, respectively, were incorrectly evaluated. Equation (7) may indeed be rewritten $\sigma_{\mathrm{d}}^{2}=r^{2} \sigma_{\mathrm{H}}^{2}+r^{2} \sigma_{\mathrm{T}}^{2}$. The two interpretations of $r$ are in fact algebraically equivalent, as it will become apparent in Eq. (9). It is shown in Fig. 1 that the inclusion of $r$ is necessary to ensure that the $\chi^{2}$ variable of Eq. (1) really follows a $\chi^{2}$ distribution. The correctness of our statistical treatment is also illustrated in Sect. 6 in relation with Fig. 11, where the rate of false detections is exactly the one expected.

After some algebra, it is then possible to show that

$S \equiv\left(\begin{array}{ccc}r_{\alpha}^{2}\left(\sigma_{\alpha \mathrm{H}}^{2}+\sigma_{\alpha \mathrm{T}}^{2}\right) & \rho_{\mathrm{H}} r_{\alpha} r_{\delta} \sigma_{\alpha \mathrm{H}} \sigma_{\delta \mathrm{H}} \\ \rho_{\mathrm{H}} r_{\alpha} r_{\delta} \sigma_{\alpha \mathrm{H}} \sigma_{\delta \mathrm{H}} & r_{\delta}^{2}\left(\sigma_{\delta \mathrm{H}}^{2}+\sigma_{\delta \mathrm{T}}^{2}\right)\end{array}\right)$.

It must be noted that in the derivation of Eq. (9), to ensure that the determinant of the $S$ matrix is always positive (ensuring the

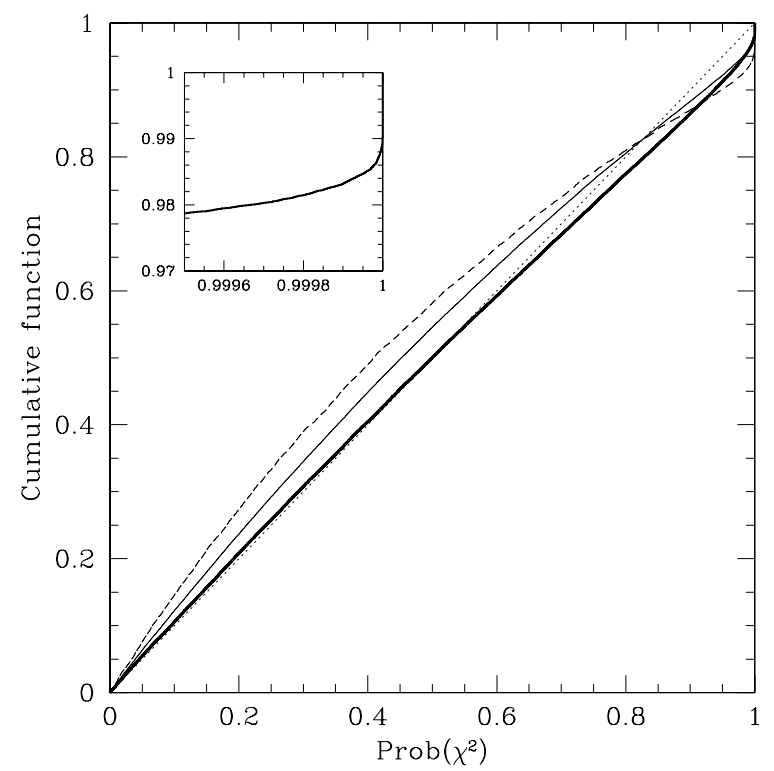

Fig. 1. Comparison of the cumulative frequency distribution of the $\chi^{2}$ values from Eq. (1) evaluated with (thick solid line) and without (thin solid line) the correction term $r$ from Eq. (7). Note how well the $r$-corrected distribution follows the diagonal (dotted line), as it should in the absence of binaries. The brightest stars with $V_{\mathrm{T}}<7$ were not corrected, and correspond to the dashed line which deviates markedly from the diagonal. The insert is a zoom on the upper right portion of the diagram, for the complete sample of 103134 stars.

positiveness of the $\chi^{2}$ as defined in Eq. (1)), the correlation term $\rho_{\mathrm{H}} \sigma_{\alpha \mathrm{H}} \sigma_{\delta \mathrm{H}}$ in Eq. (4) must be replaced by $\rho_{\mathrm{H}} r_{\alpha} r_{\delta} \sigma_{\alpha \mathrm{H}} \sigma_{\delta \mathrm{H}}$. This modification follows naturally from the realisation that the $r_{\alpha}$ and $r_{\delta}$ factors account for the possible mis-evaluation of the internal errors $\sigma_{\alpha \mathrm{H}}, \sigma_{\delta \mathrm{H}}$.

\section{Results: candidate proper-motion binaries}

The confidence level of 0.9999 on the $\chi^{2}$ probability to flag a star as binary has been set to minimise the fraction of false detections. This threshold translates into $\chi^{2}=18.4206$ (with two degrees of freedom). The insert in Fig. 1 reveals that 1734 stars out of 103134 have $\operatorname{Prob}\left(\chi^{2}\right)>0.9999^{2}$ representing $1.68 \%$ of the total sample, whereas the expected number of false detections is $0.01 \%$ of 103134 , or only 10 stars. In the remainder of this paper, stars fulfilling the condition $\operatorname{Prob}\left(\chi^{2}\right)>0.9999$ will be called (candidate) " $\Delta \mu$ binaries". Among these 1734 candidate binaries, 729 were already flagged in the Hipparcos DMSA as exhibiting a non-uniform proper motion (i.e., $162 \mathrm{DMSA} / \mathrm{G}$ solutions with 9 parameters: the 5 usual astrometric parameters, plus 4 parameters describing the first and second derivatives of the proper motion, and 567 DMSA/G solutions with 7 parameters, corresponding to a linear variation of the proper motion).

Several more proper-motion binaries actually exist in the Hipparcos Catalogue, since 1831 among the $2560 \mathrm{DMSA} / \mathrm{G}$ stars present in our sample were not flagged as binaries by the $\chi^{2}$ test. This is in part a consequence of the fact that the proper motion provided by the Hipparcos Catalogue for DMSA/G stars already includes some correction for the orbital motion, so that the difference between the long-term (i.e. Tycho-2) and short-term (i.e. Hipparcos) proper motions is not necessarily significant. In total, we thus identify 3565 candidate

\footnotetext{
2 In the remainder of this paper, $\operatorname{Prob}\left(\chi^{2}\right)$ denotes the left-sided cumulative $\chi^{2}$ probability, i.e., from 0 to $\chi^{2}$.
} 
Table 2. The first 20 entries in the list of $3565 \mathrm{DMSA} / \mathrm{G}$ or $\Delta \mu$ binaries at the 0.9999 confidence level. The full list is only available electronically at the CDS, Strasbourg. $\chi^{2}$ and $\operatorname{Prob}\left(\chi^{2}\right)$ characterise the significance of the difference between the Hipparcos and Tycho-2 proper motions (see Eq. (1)). The "DMSA" column refers to the Double and Multiple Systems Annex of the Hipparcos Catalogue (ESA 1997): "5" stands for single-star (5-parameter) solution, "7" and "9" for accelerated proper motions (7- and 9-parameter solutions), and "X" stands for stochastic solution. The columns labelled "Spectrosc. bin." provide information about the possible spectroscopic-binary nature of the star, using data from the $S_{\mathrm{B} 9}$ catalogue (Pourbaix et al. 2004), the Geneva-Copenhagen survey of F and G dwarfs in the solar neighbourhood (Nordström et al. 2004), or the KIII-MIII sample of Famaey et al. (2005). In those columns, "B" or "b" means that the star is flagged as a spectroscopic binary in these catalogues (with $\operatorname{Prob}\left[\chi^{2}\left(V_{\mathrm{r}}\right)\right] \leq 0.001$ and $0.001<\operatorname{Prob}\left[\chi^{2}\left(V_{\mathrm{r}}\right)\right] \leq 0.01$, respectively), "O" that a spectroscopic orbit is available, "c" that it is present in the catalogue but that it is not flagged as binary. The column "Remark" provides a comment relating to specific properties of the star: long-period variable (LPV); barium star (Ba); triple system (triple) or candidate triple system (triple?); suspected binary with low-mass companion (sB-lm?); spurious proper-motion binary due to perspective acceleration (perspective acc.).

\begin{tabular}{|c|c|c|c|c|c|c|c|}
\hline \multirow[t]{2}{*}{ HIP } & \multirow[t]{2}{*}{$\chi^{2}$} & \multirow[t]{2}{*}{$\operatorname{Prob}\left(\chi^{2}\right)$} & \multirow[t]{2}{*}{ DMSA } & \multicolumn{3}{|c|}{ Spectrosc. bin. } & \multirow[t]{2}{*}{ Remark } \\
\hline & & & & $S_{\mathrm{B}^{9}}$ & Gen-Cop & $\overline{\mathrm{K}-\mathrm{M}}$ & \\
\hline 62 & 3.70 & 0.84276 & 7 & - & - & - & \\
\hline 68 & 573.65 & 1.00000 & 7 & - & - & - & \\
\hline 93 & 32.19 & 1.00000 & 5 & - & B & - & \\
\hline 104 & 9.48 & 0.99126 & 7 & - & - & - & \\
\hline 171 & 1695.38 & 1.00000 & $X$ & $\mathrm{O}$ & B & - & \\
\hline 290 & 91.51 & 1.00000 & 5 & - & B & - & \\
\hline 305 & 42.75 & 1.00000 & 7 & - & $\mathrm{c}$ & - & \\
\hline 329 & 37.94 & 1.00000 & 5 & - & - & - & \\
\hline 340 & 8.28 & 0.98408 & 9 & - & - & - & \\
\hline 356 & 43.03 & 1.00000 & 5 & - & B & - & \\
\hline 359 & 105.95 & 1.00000 & 5 & - & - & - & \\
\hline 493 & 3.74 & 0.84589 & 7 & - & $\mathrm{c}$ & - & sB-lm? \\
\hline 611 & 11.68 & 0.99708 & 9 & - & - & - & \\
\hline 626 & 21.38 & 0.99998 & 5 & - & - & - & \\
\hline 646 & 2.98 & 0.77418 & 7 & - & - & - & \\
\hline 648 & 5.74 & 0.94337 & 9 & - & - & - & \\
\hline 695 & 5.10 & 0.92177 & 7 & - & - & - & \\
\hline 727 & 43.81 & 1.00000 & 5 & - & - & - & \\
\hline 741 & 13.24 & 0.99867 & 7 & - & - & - & \\
\hline 765 & 19.10 & 0.99993 & $\mathrm{X}$ & - & - & - & \\
\hline
\end{tabular}

proper-motion binaries (=1831 DMSA/G stars not reaching the 0.9999 confidence level +1734 stars above that level; among the latter, there are $1734-729=1005$ candidate proper-motion binaries not already flagged as DMSA/G). These 3565 objects are listed in Table 2, only available in electronic form at the CDS, Strasbourg. This number is close - albeit not identical to the 3783 candidate binaries (among which 1161 are not DMSA/G) identified by Makarov \& Kaplan (2005) in their Tables 1 and 2. Before comparing our results with those of Makarov \& Kaplan (2005), it should be mentioned that among DMSA/X (stochastic) solutions, the fraction of candidate proper-motion binaries (45/1403 or $3.2 \%)$ is only slightly larger than among the full sample (1.68\%), suggesting that long-period binaries are not the major cause of stochasticity.

\section{Comparison with Makarov \& Kaplan results}

Makarov \& Kaplan (2005) adopted a very simple criterion to flag a star as a candidate proper-motion binary, namely that the difference between the Hipparcos and Tycho-2 proper motions exceeds 3.5 times their root-mean square error for at least one of the two components. For a Gaussian distribution, this threshold corresponds to a fraction of false detections of $2 \times 4.7 \times$ $10^{-4} \sim 10^{-3}$, or 100 stars in a sample of $10^{5}$. Our threshold is a factor of 10 more stringent. This is immediately apparent in Fig. 2, which compares the $\chi^{2}$ values for Makarov \& Kaplan's candidate proper-motion binaries (shaded histogram) with our 0.9999 confidence level corresponding to $\chi^{2} \geq 18.4206$. A 0.999 confidence level (corresponding to the false detection rate of Makarov \& Kaplan) translates into $\chi^{2} \geq 13.8155$ (for two degrees of freedom), which indeed matches the left edge of Makarov \& Kaplan's $\chi^{2}$ distribution of their candidate proper motion binaries.

It is in the range $12 \leq \chi^{2} \leq 25$ that the two sets differ, as may in fact be expected (Fig. 2): in that range, 316 stars were flagged as proper-motion binaries by Makarov \& Kaplan, but not by us, while 138 were flagged by us and not by Makarov \& Kaplan. This difference mainly results from the different criteria used in these two studies: if the difference between the Hipparcos and Tycho proper motions is about $3.5 \sigma$ for one component only, the corresponding $\chi^{2}$ will only amount to $3.5^{2}=12.25$, well below the detection threshold used in the present work. Conversely, the difference in each component might be slightly smaller than $3.5 \sigma$, but the combined $\chi^{2}$ (Eqs. (1) and (9)) may be larger than 18.4 (for instance $3.1^{2}+3.1^{2}=19.2$ ). A good example thereof is HIP 115929 not flagged by Makarov \& Kaplan, despite its $\chi^{2}$ of 26.12 yielding $\operatorname{Prob}\left(\chi^{2}\right)=0.9999978$ in our method. With $\mu_{\alpha *}(\mathrm{HIP})=-5.29 \pm 1.08 \mathrm{mas}^{-1}$ and $\mu_{\alpha *}($ Tycho -2$)=-10.2 \pm 1.2$ mas $\mathrm{y}^{-1}$, the difference in $\mu_{\alpha *}$ amounts to $\left|\Delta \mu_{\alpha^{*}}\right| /\left[\epsilon^{2}(\mathrm{HIP})+\epsilon^{2}(\text { Tycho }-2)\right]^{1 / 2}=3.0$, and with $\mu_{\delta}(\mathrm{HIP})=36.52 \pm 0.81 \mathrm{mas}^{-1}, \mu_{\delta}($ Tycho -2$)=32.1 \pm$ $1.1 \mathrm{mas}^{-1}$, the difference in $\delta$ amounts to $\left|\Delta \mu_{\delta}\right| /\left[\epsilon^{2}(\mathrm{HIP})+\right.$ $\epsilon^{2}($ Tycho -2$\left.)\right]^{1 / 2}=3.2$. An additional factor acting in the same direction is that, with a Tycho- 2 magnitude $V_{\mathrm{T}}=9.01$, HIP 115929 belongs to the magnitude range where the $r_{\alpha}$ and $r_{\delta}$ parameters (Table 1) have the largest impact on the $\chi^{2}$ value, increasing it by about 20\%. Incidentally, HIP 115929 is also flagged as a $\Delta \mu$ binary in the DMUBIN database (Wielen et al. 1999). 


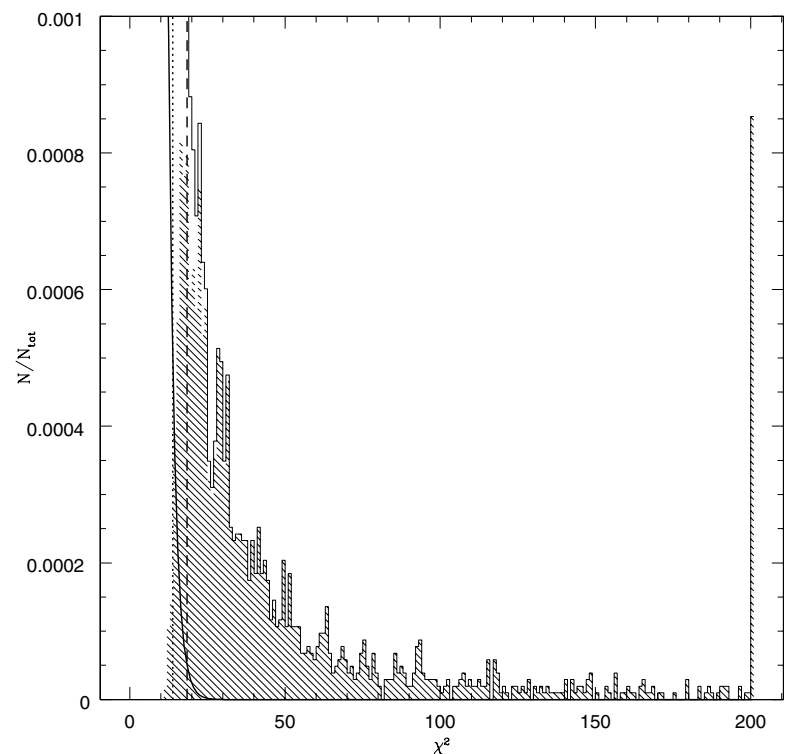

Fig. 2. Tail of the $\chi^{2}$ distribution containing the proper-motion binaries, located to the right of the vertical dashed line, corresponding to the threshold used in the present work $\left[\operatorname{Prob}\left(\chi^{2}\right) \geq 0.9999\right.$, translating into $\chi^{2} \geq 18.4$ for two degrees of freedom]. The candidate proper-motion binaries from Makarov \& Kaplan (2005) are identified by the shaded histogram. They are mostly located to the right of the dotted line, corresponding to $\operatorname{Prob}\left(\chi^{2}\right) \geq 0.999$, or $\chi^{2} \geq 13.8$ (for two degrees of freedom). The solid line is the theoretical $\chi^{2}$ distribution with two degrees of freedom expected in the case of a pure single-star sample.

\section{Comparison with other samples of known binaries}

Our list of candidate proper-motion binaries identified in Sect. 3 can be confronted with several independent data sets available, namely (i) the spectroscopic binaries from the Ninth Catalogue of Spectroscopic Binary Orbits $\left(S_{\mathrm{B}^{9}}\right.$; Pourbaix et al. 2004); (ii) barium stars listed by Lü et al. (1983); (iii) the radial-velocity standard deviation available from the Geneva-Copenhagen survey of $\mathrm{F}$ and $\mathrm{G}$ dwarfs in the solar neighbourhood (Nordström et al. 2004), and (iv) the radial-velocity standard deviation available from the Famaey et al. (2005) catalogue of K and M giants in the solar neighbourhood. The latter two data sets are subsamples resulting from the major effort to collect radial velocities for about 45000 Hipparcos "survey" stars later than F5 (Udry et al. 1997), using the CORAVEL spectrovelocimeter (Baranne et al. 1979). These data will, among other things, enable us to check whether the proper-motion binaries are indeed found in the orbital-period range predicted by Makarov \& Kaplan (2005) an important observational test for the method, not attempted before. As a general remark applying to the remainder of this paper, we stress that the orbital inclination somewhat blurs the comparison between proper-motion and spectroscopic diagnostics, since proper-motion binaries seen close to pole-on will not be detected as spectroscopic binaries.

\section{1. $S_{\mathrm{B}^{9}}$ entries}

At the time of our query, the Ninth Catalogue of Spectroscopic Binary Orbits $\left(S_{\mathrm{B}^{9}}\right.$; Pourbaix et al. 2004) holds orbits for 2386 systems, among which 1320 have a Hipparcos and Tycho-2 entry. The period distribution for those stars is presented in Fig. 3, along with the frequency of stars for which the Hipparcos and Tycho-2 proper motions differ at confidence levels of 0.9999

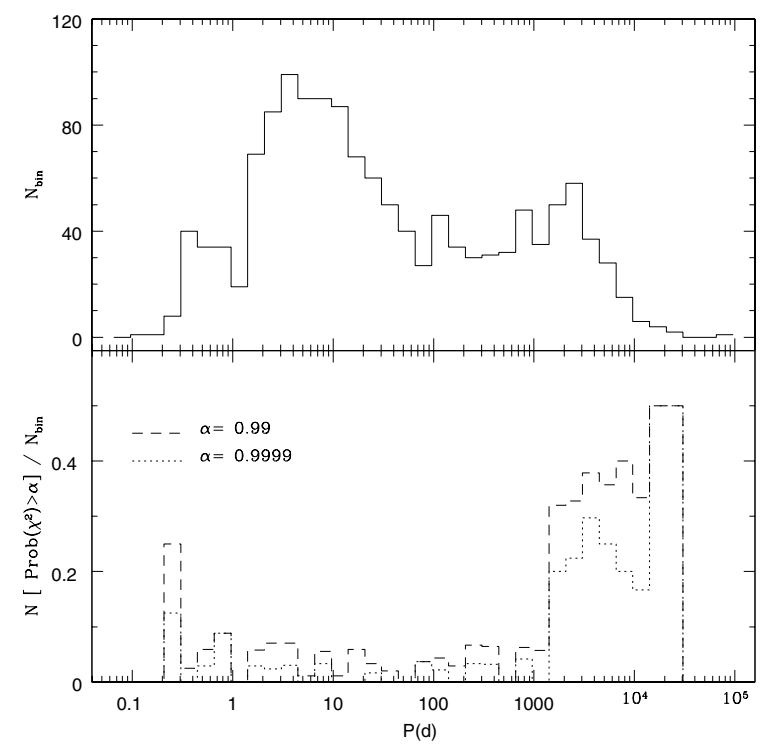

Fig. 3. Upper panel: orbital-period distribution for stars from the $S_{\mathrm{B} 9}$ catalogue. Lower panel: fraction of stars in a given bin for which the Hipparcos and Tycho-2 proper motions differ at confidence levels of 0.9999 (dotted line) and 0.99 (dashed line).

and 0.99. Careful inspection of the data shows that the lower limit on period for efficient $\Delta \mu$ binary detection is located at $1500 \mathrm{~d}$ (see also Fig. 4). Proper-motion binaries with periods up to $30000 \mathrm{~d}$ have been detected, corresponding to the longest periods present in the $S_{\mathrm{B}^{9}}$ catalogue. Among the long-period proper-motion binaries detected, all eccentricities are equally well represented. Figure 4 presents an eccentricity-period diagram for $S_{\mathrm{B}^{9}}$ stars with parallaxes larger than 10 mas. Note that almost all such objects with $P>1000 \mathrm{~d}$ have been flagged as either DMSA/G or $\Delta \mu$ binaries. On the other hand, $\Delta \mu$ or DMSA/G binaries with orbital periods shorter than about $400 \mathrm{~d}$ are (candidate) triple systems (see the discussion below and Tables 3 and 4).

Makarov \& Kaplan (2005) have presented a detailed analysis of the relationship between the system's orbital period and eccentricity, and the probability that such a system be either detected as a proper-motion binary (i.e., with $\Delta \mu$ significantly different from 0), or as DMSA/G (i.e., with an acceleration term in the proper motion, $\dot{\mu})$. They argued that systems for which the latter effect is stronger will be mainly found in the period range 3 to $6 \mathrm{yr}$ (1000 to $2000 \mathrm{~d}$ ), whereas systems detectable as $\Delta \mu$ binaries, but not as $\dot{\mu}$ binaries will be mainly found among binaries with even longer periods. This is exactly what is seen in Fig. 4. Systems solely detected as DMSA/G and not as propermotion binaries are mainly restricted to the short-period side of the $P>1000 \mathrm{~d}$ range.

As shown theoretically by Makarov \& Kaplan (2005), systems ought to have a parallax large enough to be detected as proper-motion binaries. Figure 5 illustrates the incidence of the parallax on the detection efficiency: for parallaxes between 10 and 25 mas, about $50 \%$ of the $S_{\mathrm{B}^{9}}$ stars with periods larger than $1500 \mathrm{~d}$ are detected, and that fraction becomes larger than $80 \%$ above 25 mas. Among the 69 systems with $P>1500 \mathrm{~d}$ and parallaxes in excess of 10 mas, 28 remain undetected, either as DMSA/G or as proper-motion binaries. A closer look at those systems reveals however that 15 of them have confidence levels in excess of 0.9 , and may in fact be considered as proper-motion binaries. There is no reason to expect that all these 69 binaries should be detected by the $\Delta \mu$ method, as at 10 mas the detection 


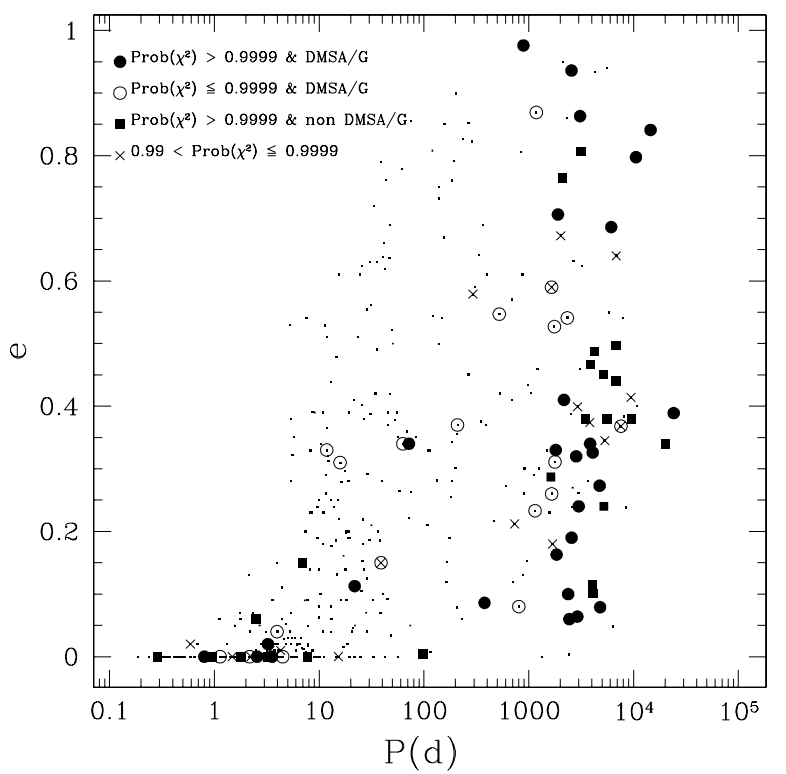

Fig. 4. Eccentricity-period diagram for stars from the $S_{\mathrm{B}^{9}}$ catalogue with parallaxes larger than 10 mas. Symbols are as follows: large filled symbols: proper-motion binaries detected at the 0.9999 confidence level (and flagged as DMSA/G: large filled circles; not flagged as DMSA/G: large filled squares); open circles: systems flagged as DMSA/G but not fulfilling the 0.9999 confidence level; crosses: systems not flagged as DMSA/G, lying in the range $0.99<\operatorname{Prob}\left(\chi^{2}\right)<0.9999$.

efficiency of the method is still below maximum (as shown in Fig. 5 and further discussed in Sect. 6 on a much larger sample). Nevertheless, we note that among the 13 remaining undetected cases, 8 might be explained by one of the following reasons:

- the spectroscopic orbit is of poor quality (HIP 108473) ${ }^{3}$;

- the system has two visible spectra (SB2 or composite spectrum), so that the photocentre is not very different from the centre of mass and no orbital motion can be detected (HIP 2563, HIP 23016, HIP 73449, HIP 114576, HIP 111528);

- the system is a triple or multiple (HIP 20430, HIP 114576);

- the system is eccentric and was observed by Hipparcos at a slowly varying orbital phase (HIP 106786; Gontcharov \& Kiyaeva 2002).

As opposed to the situation just considered (long-period binaries not detected as $\Delta \mu$ binaries), systems with $P<400 \mathrm{~d}$ should not have been detected as proper-motion binaries. Unless, that is, the short-period spectroscopic orbit is in fact the inner orbit of a wider pair involving a third component. A systematic search for a possible third star in these systems has been performed in the Multiple Star Catalogue (Tokovinin 1997) and in the Fourth Catalog of Interferometric Measurements of Binary Stars (Hartkopf et al. 2001), which includes all published measurements of binary and multiple star systems obtained by highresolution techniques (speckle interferometry, photoelectric occultation timing, etc.), as well as negative examinations for duplicity. Table 3 lists the 16 short-period $(P<400 \mathrm{~d}) \mathrm{DMSA} / \mathrm{G}$ or $\Delta \mu$ systems with firm evidence for being triple or quadruple

${ }^{3}$ It must be mentioned that HIP 108473 , not flagged as a $\Delta \mu$ binary when comparing Hipparcos and Tycho-2 proper motions, is nevertheless flagged as such when comparing Hipparcos proper motions to the ones computed from the Boss General Catalogue (Boss et al. 1937; Wielen et al. 1999).

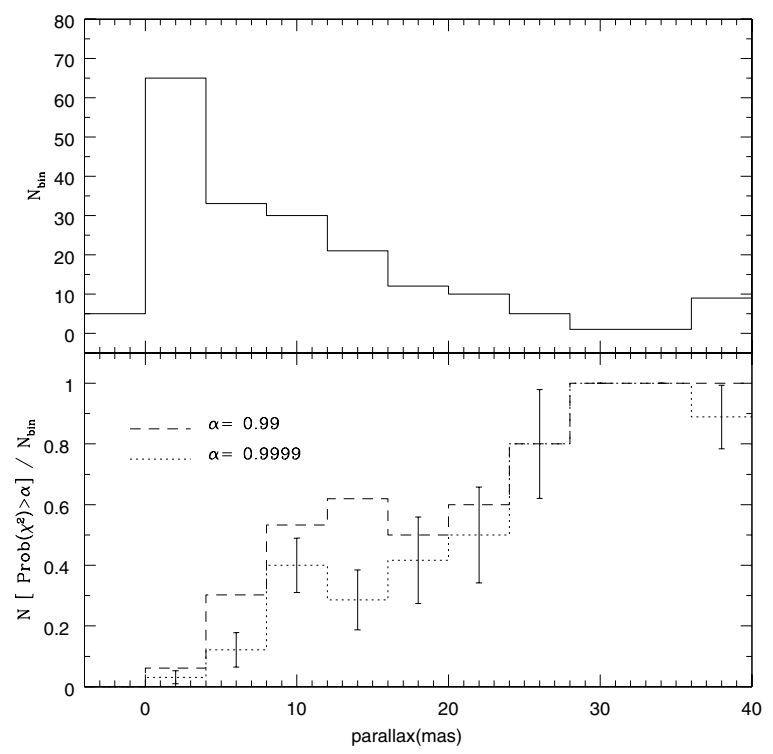

Fig. 5. Upper panel: parallax distribution of $S_{\mathrm{B}^{9}}$ stars with orbital periods larger than $1500 \mathrm{~d}$. Lower panel: fraction of those $S_{\mathrm{B}^{9}}$ stars detected as proper-motion binaries at confidence levels $\alpha$ of 0.99 and 0.9999 . For parallaxes between 10 and 25 mas, about $50 \%$ of the $S_{\mathrm{B}}$ stars with periods larger than $1500 \mathrm{~d}$ are detected, and that fraction becomes larger than $80 \%$ above 25 mas.

hierarchical systems. It is remarkable that about half of the systems (more precisely $16 / 39$ or $41 \%$ ) that are suspected of hosting a third star from the proper-motion analysis indeed turn out to be triple after close scrutiny. The case of HIP 35487 (R CMa) is exemplary in that respect: a detailed analysis of a centurylong eclipse timing of this system indeed revealed the presence of a third star (Ribas et al. 2002).

Another remarkable case is HIP 72939, flagged as a DMSA/G system but with a period of $3.55 \mathrm{~d}$ listed in $S_{\mathrm{B}^{9}}$. After the present analysis was completed, Fekel et al. (2006) obtained a spectroscopic orbit for a third, outer companion, with a period of $1641 \mathrm{~d}$. The revised Hipparcos proper motion is now within $1 \sigma$ of the Tycho- 2 one.

This proportion of $41 \%$ triple systems among short-period $S_{\mathrm{B}^{9}}$ binaries flagged as DMSA/G or $\Delta \mu$ binaries is much larger than expected from random selection, since Halbwachs et al. (2003) find on average 1 triple system for 25-30 systems (3 to $4 \%$ ) with F7-K primaries and $P<10$ yr. It therefore seems reasonable to suggest that the remaining 23 shortperiod $(P<400 \mathrm{~d})$ DMSA/G or $\Delta \mu$ systems will turn out to be triple systems as well, despite the current lack of evidence. They are listed in Table 4. A few stars should perhaps be removed from this list of candidate triple systems: HIP 21673, HIP 52444, HIP 68523, and HIP 77911, whose orbital elements are in fact considered doubtful (hence, they might not be short-period systems after all).

HIP 77911 deserves further discussion, as it is an outlier in the eccentricity - period diagram, with $P=1.26 \mathrm{~d}$ and $e=0.61 \pm 0.2$ (Levato et al. 1987, who consider their orbit as very marginal, however). A visible companion was found by Kouwenhoven et al. (2005) using the ADONIS adaptive optics system on the ESO $3.6 \mathrm{~m}$ telescope, but it is about $8^{\prime \prime}$ away, and is thus unlikely to be responsible for the DMSA/G nature of the primary star. Either the orbital period of HIP 77911 is in error, or the outlying position of HIP 77911 in the eccentricity - period diagram results from eccentricity pumping by a third star in the 
Table 3. $S_{\mathrm{B}^{9}}$ systems with $P<400 \mathrm{~d}$, flagged as DMSA/G or $\Delta \mu$ systems, and with firm evidence for being triple or quadruple.

\begin{tabular}{|c|c|c|c|c|}
\hline HIP & DMSA/G & $P(\mathrm{~d})$ & $e$ & $\overline{\text { Remark }}$ \\
\hline 24156 & 7 & 0.42 & 0.0 & SB2; triple system (Goecking et al. 1994; Kim et al. 2003) \\
\hline 30630 & 5 & 6.99 & 0.15 & $\begin{array}{l}\text { SB2 resolved; suspected triple as the systemic velocity seems to } \\
\text { be variable }\end{array}$ \\
\hline \multirow[t]{2}{*}{35487} & 7 & 1.13 & 0.0 & $\begin{array}{l}\text { SB2 (F1V + G8IV-V); triple system detected by light-travel } \\
\text { time effect (Ribas et al. 2002) }\end{array}$ \\
\hline & 7 & $3.4 \times 10^{4}$ & 0.49 & \\
\hline 42542 & 9 & 48.72 & 0.11 & Triple system (Hartkopf et al. 2001; Pourbaix et al. 2004) \\
\hline \multirow[t]{2}{*}{49136} & 5 & 0.28 & 0.0 & $\begin{array}{l}\text { Quadruple system: two close pairs separated by } 0.147 \text { arcsec on } \\
\text { the sky, }\end{array}$ \\
\hline & 5 & 0.81 & 0.0 & in an orbit of period $7 \times 10^{3} \mathrm{~d}($ Tokovinin 1997$)$ \\
\hline \multirow[t]{2}{*}{60129} & 7 & 71.9 & 0.34 & Triple system (Hartkopf et al. 1992) \\
\hline & 7 & 4792 & 0.079 & \\
\hline 72939 & 7 & 3.55 & 0.0 & $\begin{array}{l}\text { Triple system (Mayor \& Mazeh 1987; Tokovinin 1997; Fekel } \\
\text { et al. 2006) }\end{array}$ \\
\hline \multirow[t]{2}{*}{84886} & 7 & 21.82 & 0.1126 & Triple system (Griffin \& Cornell 1997; Tokovinin 1997) \\
\hline & 7 & 2909 & 0.06 & \\
\hline 86187 & 5 & 2.50 & 0.06 & $\begin{array}{l}\text { Triple system: third star found at about } 0.3 \text { arcsec; systemic } \\
\text { velocity of the inner pair is variable (Carquillat et al. 1976; } \\
\text { Hartkopf et al. 2001) }\end{array}$ \\
\hline 87655 & 7 & 0.80 & 0 & $\begin{array}{l}\text { Triple system: the third star is in an orbit of period } 8.9 \mathrm{yr} \text { around } \\
\text { the close pair (Söderhjelm 1999; Tokovinin 1997) }\end{array}$ \\
\hline \multirow[t]{2}{*}{88848} & $\mathrm{X}$ & 1.81 & 0.0 & Triple system (Fekel et al. 2005) \\
\hline & $\mathrm{X}$ & 2092.2 & 0.765 & \\
\hline 90312 & 5 & 8.80 & 0 & SB2 (G2IV + K2IV); suspected non single in Hipparcos \\
\hline 98954 & 9 & 198.7 & 0.05 & A third component at $2.3^{\prime \prime}$ (Tokovinin 1997) \\
\hline 103987 & 9 & 377.82 & 0.086 & $\begin{array}{l}\text { Triple system (third star at } 0.132^{\prime \prime} \text { with an estimated period of } \\
14 \text { yr; Mason et al. 2001) }\end{array}$ \\
\hline \multirow[t]{2}{*}{105091} & 7 & 225.44 & 0.23 & Triple system \\
\hline & 7 & 5.41 & 0.11 & \\
\hline 117712 & 5 & 7.75 & 0.0 & $\begin{array}{l}\text { Twin SB2: K3V }+\mathrm{K} 3 \mathrm{~V} \text {; triple system: third star at } 4.6^{\prime \prime} \text { in an } \\
\text { orbit of period } 9 \times 10^{4} \mathrm{~d} \text { (Tokovinin 1997) }\end{array}$ \\
\hline
\end{tabular}

Table 4. List of candidate triple systems. It consists of $S_{\mathrm{B} 9}$ systems with $P<400 \mathrm{~d}$, flagged as DMSA/G or $\Delta \mu$ systems, with no firm evidence so far of being a triple system. In column remark, "not resolved" means that no visual companion is listed in the Fourth Catalog of Interferometric Measurements of Binary Stars (Hartkopf et al. 2001).

\begin{tabular}{lllll}
\hline \hline HIP & DMSA/G & $P(\mathrm{~d})$ & $e$ & Remark \\
\hline 3362 & 7 & 2.17 & 0.0 & SB2 $(\mathrm{dM} 1 \mathrm{e}+\mathrm{dM} 1 \mathrm{e})$ \\
12716 & 5 & 0.95 & 0 & twin SB2: G6 + K0; not resolved \\
14273 & 7 & 2.73 & 0.0 & SB2 \\
18080 & 5 & 0.51 & 0.07 & W UMa binary \\
19248 & 7 & 2.56 & 0 & SB2 \\
23657 & 5 & 1.58 & 0.06 & SB2 (B5V + B5V); unlikely to be $\Delta \mu$ binary because parallax is very small \\
34630 & 7 & 20.63 & 0.296 & SB2 \\
45080 & 7 & 6.74 & 0.18 & \\
50097 & 5 & 3.24 & 0.0 & \\
53295 & 7 & 15.83 & 0.31 & not resolved \\
70931 & 9 & 11.82 & 0.33 & \\
89601 & 7 & 4.48 & 0.00 & not resolved \\
95028 & 7 & 208.8 & 0.37 & not resolved \\
98351 & 7 & 62.88 & 0.34 & not resolved \\
100142 & 7 & 2.98 & 0.07 & twin SB2: B2V + B2V; not resolved \\
104987 & 5 & 98.82 & 0.0 & SB2 \& visual binary (G2III + A5V) \\
105406 & 7 & 3.24 & 0.02 & SB2 (F8V + K6V); not resolved \\
110514 & 7 & 6.72 & 0.178 & twin SB2: F8IV + F7IV \\
114639 & 7 & 3.96 & 0.04 & SB2: K1IV + F8V; not resolved \\
& & & & \\
Weak candidates & & & & \\
21673 & 9 & 38.95 & 0.15 & not resolved; orbital elements considered "marginal" \\
52444 & 7 & 51.55 & 0.0 & orbital elements based on small number of data points \\
68523 & 9 & 207.36 & 0.55 & not resolved; orbital elements uncertain \\
77911 & 7 & 1.26 & 0.61 & either triple system as suggested by eccentricity pumping or doubtful orbital elements (see text) \\
\hline
\end{tabular}




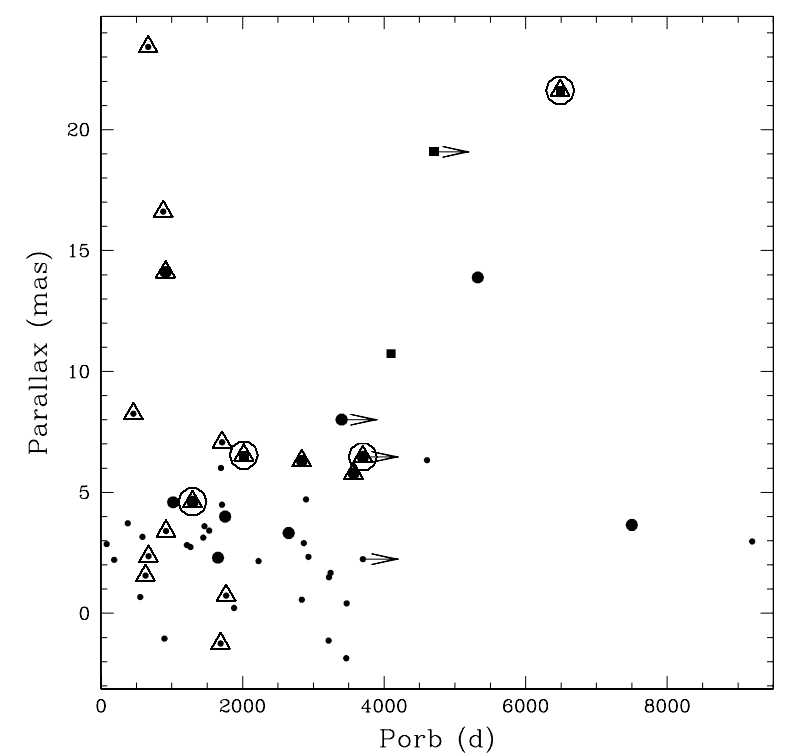

Fig. 6. Barium stars with known orbital periods and parallaxes. Objects flagged as $\Delta \mu$ binaries are represented by filled squares if $\operatorname{Prob}\left(\chi^{2}\right)>$ 0.9999 (flag 4 in Table 5), and by large filled circles if $0.9<\operatorname{Prob}\left(\chi^{2}\right) \leq$ 0.9999 (flag 5). DMSA/G binaries are represented by large open circles. Large open triangles correspond to binaries where an astrometric orbit could be derived (flag 3) or at least an astrometric signature of binarity has been detected (flag 2). Arrows denote stars with only a lower limit available on the period. Small filled circles are barium stars that were not flagged as binaries by any of the astrometric methods to detect duplicity.

system (Mazeh \& Shaham 1979; Mazeh 1990), the same which is in fact responsible for the accelerated proper motion.

Considering the 16 firm and the 19 suspected triple systems, the frequency of triple systems amount to $35 / 1320$ (or $2.6 \%$ ) among the $S_{\mathrm{B}^{9}}$ systems considered in the present analysis. This frequency is consistent with the frequency of triple systems reported by Halbwachs et al. (2003). To conclude this discussion, it is interesting to remark that several of the candidate triple stars listed in Table 4 are so-called "twins", i.e., short-period $(P<10$ d) SB2 systems with components of (almost) identical spectral types. It has been argued that many twins are in fact members of higher-multiplicity systems (Tokovinin 2000, see however Halbwachs et al. (2003) who do not share this opinion).

\subsection{Barium stars}

Barium stars represent a very interesting control sample for the binary-detection method discussed in this paper, since they are known to be all binaries with periods in the range $80-10^{4} \mathrm{~d}$ (Jorissen 2003). Therefore, the detection rate of $\Delta \mu$ binaries among barium stars provides a hint on the detection biases. The catalogue of Lü et al. (1983) contains 163 bona fide barium stars with an HIP entry (excluding the supergiants HD 65699 and HD $206778=\epsilon$ Peg). This number drops to 156 after removing DMSA/C and DMSA/O entries. Only $9 \Delta \mu$ binaries are detected among barium stars at the confidence level 0.9999 (HIP 13055, $58948,65535,71058,97613,98431,104732,105294,112821)$, 5 of which being moreover flagged as DMSA/G. This rather small number of detections may appear surprising at first sight, since astrophysical considerations require all barium stars to be binaries (McClure et al. 1980; McClure 1983; McClure \& Woodsworth 1990; Jorissen 2003). All but 12 barium stars have

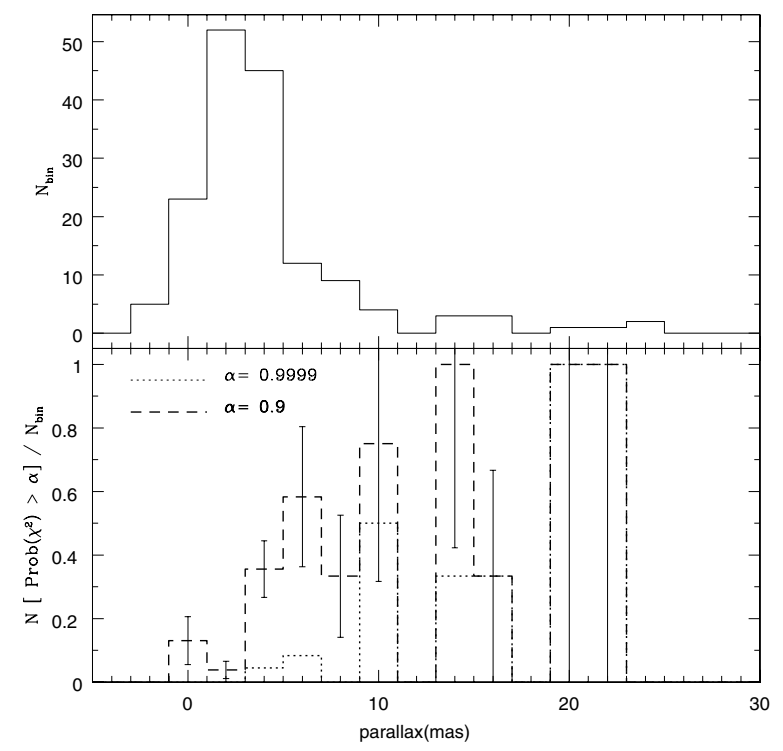

Fig. 7. Same as Fig. 5, but for barium stars.

parallaxes smaller than 10 mas, though, which makes the detection of $\Delta \mu$ binaries among barium stars very inefficient, as discussed in Sects. 5.1 and 5.3. Among these 12 barium stars with parallaxes larger than 10 mas, 6 are indeed detected as $\Delta \mu$ binaries at the 0.9999 confidence level.

The present astrometric method complements several previous studies devoted to the detection of binaries among barium stars, using various astrometric and spectroscopic methods. Since these studies are scattered in the literature, it is useful to collect their results in one single table (Table 5) and figure (Fig. 6). Because the efficiency of the astrometric methods to detect binaries depends on the parallax, Table 5 has been split in two parts, for barium stars with parallaxes larger or smaller than 5 mas. This 5 mas threshold contrasts with the 20 mas threshold adopted in Sect. 6. A lower threshold for barium stars is motivated by their scarcity at larger parallaxes (Fig. 7). To reach a detection efficiency of proper-motion binaries of the order of 30 to $50 \%$ around 5 mas we relax the confidence level to 0.9 (see the discussion relative to Fig. 11 in Sect. 6; compare with Fig. 7). Objects flagged as $\Delta \mu$ binaries by this less stringent criterion have a separate flag in Table 5.

The flags listed in Table 5, referring to various astrometric methods, deserve more detailed explanations:

- Flag 2: The presence of an orbital motion in the astrometric signal may be checked by using trial orbital periods and assuming a circular orbit (for details, see Sect. 1 and Pourbaix 2004), even though the spectroscopic orbit is not available. This method has been applied to barium stars by Jorissen et al. (2004, 2005), and works best when $\varpi>5$ mas and $100 \leq P(\mathrm{~d}) \leq 4000$ (see open triangles in Fig. 6);

- Flag 3: An astrometric orbit constrained by the spectroscopic orbital elements could be derived (Pourbaix \& Jorissen 2000; Jancart et al. 2005; open triangles in Fig. 6);

- Flags 4, 5: $\Delta \mu$ binaries, most efficiently detected in the period range 1500-10000 d. Flag 4 - detection with a confidence level of 0.9999 (filled squares in Fig. 6); flag 5 - with a confidence of 0.9 (large filled circles in Fig. 6);

- Flag 6: Binaries detected from their accelerated proper motion (flagged as DMSA/G in the Hipparcos Catalogue) correspond to periods in the range of one to a few $10^{3} \mathrm{~d}$ (large open circles in Fig. 6). 
Table 5. Duplicity of barium stars with parallaxes $\varpi>5$ mas. The flags in the second column correspond to positive (or negative) detections with the following methods: (0) no radial-velocity variations detected by CORAVEL monitoring (1) spectroscopic binary (2) astrometric orbit suspected (3) astrometric orbit constrained from spectroscopic orbit (4) $\Delta \mu$ binary with $\operatorname{Prob}\left(\chi^{2}\right)>0.9999$ (5) $\Delta \mu$ binary with $0.9<\operatorname{Prob}\left(\chi^{2}\right) \leq 0.9999$ (6) accelerated proper motion (DMSA/G binary). Flags (0) and (1) are derived from Jorissen et al. (1998), Udry et al. (1998), Nordström et al. (2004); flag (2) from Jorissen et al. (2004, 2005); flag (3) from Pourbaix \& Jorissen (2000), Jancart et al. (2005); flags $(4,5)$ from the present paper; and flag (6) from the Hipparcos Catalogue DMSA. Parallaxes of barium stars flagged as $\Delta \mu$ binaries at a confidence level of 0.9999 (flag 4) have been written in bold face.

\begin{tabular}{|c|c|c|c|c|}
\hline HIP & Flags & $\begin{array}{r}P_{\text {orb }} \\
(\mathrm{d})\end{array}$ & $\begin{array}{r}\varpi \\
\text { (mas) }\end{array}$ & Rem. \\
\hline 944 & 26 & - & 7.11 & \\
\hline 4422 & - & - & 15.84 & \\
\hline 10119 & 25 & - & 8.91 & Ba dwarf \\
\hline 13055 & 12346 & 2018 & 6.54 & \\
\hline 20102 & 1 & 1694 & 6.01 & \\
\hline 31205 & 123 & 457 & 8.25 & \\
\hline 32894 & 125 & 912 & 14.11 & \\
\hline 33628 & 1256 & $>3700$ & 6.47 & \\
\hline 39426 & 05 & - & 6.34 & \\
\hline 47267 & 1256 & - & 6.73 & $\mathrm{SB}$ in Gen-Cop \\
\hline 49166 & - & - & 7.90 & \\
\hline 50805 & 123 & 667 & 23.42 & \\
\hline 53807 & 0 & - & 9.54 & \\
\hline 56130 & - & - & 5.46 & \\
\hline 56731 & 123 & 1711 & 7.07 & \\
\hline 58948 & 14 & $>4700$ & 19.08 & \\
\hline 60292 & 1235 & 3569 & 5.78 & \\
\hline 62608 & 5 & - & 6.20 & \\
\hline 65535 & 246 & - & 15.73 & \\
\hline 69176 & - & - & 8.75 & \\
\hline 71058 & 4 & - & 10.63 & \\
\hline 76425 & 15 & 5324 & 13.89 & \\
\hline 80356 & 15 & $>3400$ & 8.01 & \\
\hline 81729 & - & - & 23.35 & \\
\hline 89386 & 2 & - & 5.69 & \\
\hline 89881 & 25 & - & 9.37 & \\
\hline 93188 & 2 & - & 5.55 & \\
\hline 98431 & 4 & - & 14.06 & \\
\hline 103263 & 1 & 4606 & 6.33 & \\
\hline 104732 & 1246 & 6489 & 21.62 & \\
\hline 105881 & 12 & 2378 & 8.19 & \\
\hline 105969 & 13 & 878 & 16.61 & Ba dwarf \\
\hline 106306 & 125 & 2837 & 6.31 & \\
\hline 107818 & - & - & 7.53 & \\
\hline 112821 & 14 & 4098 & 10.74 & \\
\hline 114155 & 1 & 111 & 6.07 & \\
\hline 117585 & 25 & - & 7.08 & \\
\hline
\end{tabular}

For the sake of completeness, spectroscopic binaries (from Jorissen et al. 1998) are mentioned as well in Table 5 with flag 1 (flag 0 in the case that no radial velocity variations have been detected by CORAVEL monitoring). No flag means that the star was not detected as binary by any of the astrometric methods and it was not included in the CORAVEL survey. Figure 6 is remarkable in demonstrating quite clearly the ability of the various astrometric methods to detect the orbital motion all the way from $500 \mathrm{~d}$ to more than $5000 \mathrm{~d}$, provided that the parallax be larger than about 5 mas. The present analysis thus completes the series of papers (Jorissen et al. 1998; Pourbaix \& Jorissen 2000; Jorissen et al. 2004, 2005) devoted to the detection of binaries among barium stars. Among the 37 barium stars with parallaxes
Table 5. Continued for barium stars with $\varpi<5$ mas. Only systems for which at least one flag can be set are listed.

\begin{tabular}{rlrrl}
\hline \hline HIP & Flags & $\begin{array}{r}P_{\text {orb }} \\
\text { (d) }\end{array}$ & $\begin{array}{r}\varpi \\
\text { (mas) }\end{array}$ & Rem. \\
\hline 558 & 5 & - & 1.65 & \\
21519 & 5 & - & 4.55 & \\
21681 & 25 & - & 3.44 & \\
25452 & 15 & 2652 & 3.32 & \\
25547 & 25 & - & 3.43 & \\
27767 & 5 & - & 0.83 & \\
29740 & 123 & 1689 & -1.25 & \\
30338 & 13 & 629 & 1.56 & \\
32713 & 13 & 1768 & 0.73 & \\
34143 & 15 & 7500 & 3.65 & \\
34795 & 02 & - & 2.44 & \\
36042 & 13 & 672 & 2.36 & \\
38488 & 2 & - & 1.29 & \\
42999 & 5 & - & 0.99 & \\
47881 & 26 & - & 3.56 & \\
50259 & 5 & - & 4.38 & \\
51533 & 5 & 1754 & 4.00 & \\
52271 & 13 & 918 & 3.4 & \\
53091 & 2 & - & 2.14 & \\
53717 & 15 & 1653 & 2.30 & \\
61175 & 5 & - & 4.89 & \\
66844 & 05 & - & 3.87 & \\
88743 & 5 & - & 3.75 & \\
90316 & 2 & - & 0.56 & \\
96024 & 2 & - & 3.48 & \\
97613 & 246 & - & $\mathbf{4 . 1 0}$ & \\
97874 & 2 & - & 2.07 & \\
105294 & 246 & - & $\mathbf{3 . 4 9}$ & Ba dwarf \\
107478 & 2 & - & 3.75 & \\
107685 & 2 & - & 1.77 & \\
109662 & 5 & - & -0.10 & \\
110108 & 15 & 1019 & 4.59 & \\
113641 & 5 & - & 3.17 & \\
117607 & 1356 & 1294 & 4.61 & \\
117829 & 5 & - & 4.42 & \\
\hline & & & & \\
& & & \\
& 5 & &
\end{tabular}

larger than 5 mas, there are only 7 with no evidence of orbital motion.

\subsection{Geneva-Copenhagen survey of $F$ and $G$ dwarfs in the solar neighbourhood}

The Geneva-Copenhagen catalogue (Nordström et al. 2004) contains about $16700 \mathrm{~F}$ and $\mathrm{G}$ dwarfs of the solar neighbourhood. For 9431 of those, members of visual pairs excluded, CORAVEL radial velocities as well as Hipparcos and Tycho-2 proper motions are available. It is then possible to confront the duplicity diagnostic based on proper-motion with that based on radial velocities. The latter criterion is based on the comparison between an internal error estimate $\epsilon$ and an external error estimate provided by the standard deviation $\sigma\left(V_{\mathrm{r}}\right)$ of the radial-velocity measurements. From these and the number of observations $N\left(V_{\mathrm{r}}\right)$, the probability $\operatorname{Prob}\left(\chi_{V_{\mathrm{r}}}^{2}\right)$ that the observed radial-velocity scatter is due to measurement errors alone may be computed from $\chi_{V_{\mathrm{r}}}^{2}=\left[N\left(V_{\mathrm{r}}\right)-1\right]\left(\sigma\left(V_{\mathrm{r}}\right) / \epsilon\right)^{2}$ (Jorissen \& Mayor 1988). We adopt $1-\operatorname{Prob}\left(\chi_{V_{\mathrm{r}}}^{2}\right) \geq 0.999$ as a threshold for flagging a star with variable radial velocity, most likely due to duplicity. Note that, with the above definitions, $\operatorname{Prob}\left(\chi_{V_{\mathrm{r}}}^{2}\right)$ is the probability for an object of being single, while the proper-motion related $\operatorname{Prob}\left(\chi^{2}\right)$ is the probability for an object of being a binary. 


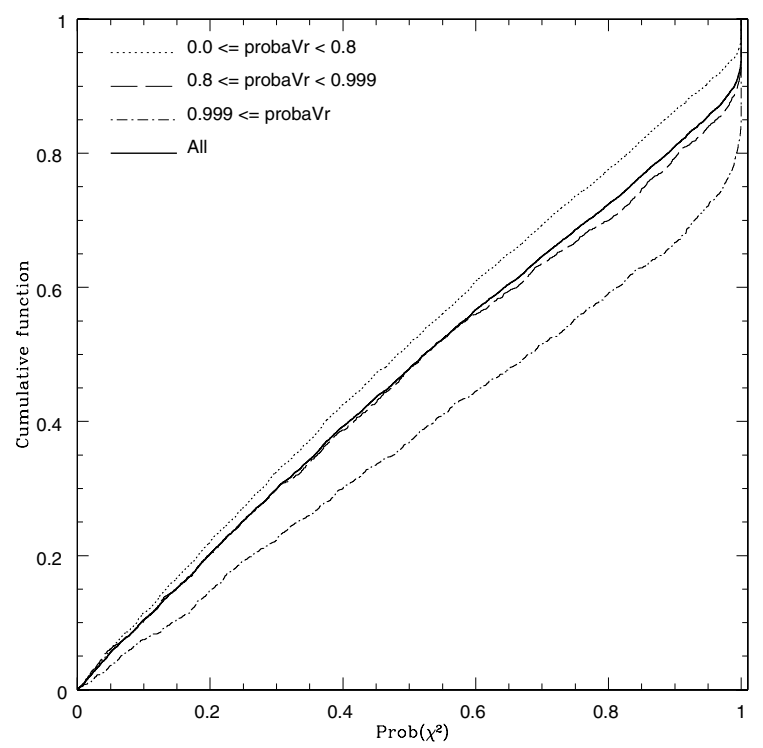

Fig. 8. Comparison of the cumulative $\operatorname{Prob}\left(\chi^{2}\right)$ (from Eq. (1)) for the Geneva-Copenhagen sample of $\mathrm{F}$ and $\mathrm{G}$ dwarfs, for different values of their radial-velocity variability probability $\left(\operatorname{probaVr} \equiv 1-\operatorname{Prob}\left(\chi_{V_{\mathrm{r}}}^{2}\right)\right.$ ). The largest fraction of proper-motion binaries is indeed found among radial-velocity binaries, having probaVr $\geq 0.999$.

As revealed by Fig. 8, the largest fraction (about 15\%, raising to $22 \%$ when adding DMSA/G systems) of proper-motion binaries indeed arises among the stars flagged as radial-velocity binaries at the $99.9 \%$ confidence level. These fractions become respectively $29.9 \pm 2.4 \%(=109 / 364)$ and $35.7 \pm 2.5 \%$ $(=130 / 364)$ when eliminating those stars with parallaxes smaller than 20 mas among which proper-motion binaries are not efficiently detected. This fraction of only $35.7 \%$ proper-motion binaries among spectroscopic binaries reflects the fact that spectroscopic and proper-motion binaries span different period ranges: $P_{\mathrm{PM}} \geq 1000 \mathrm{~d}$ for proper-motion binaries in general, and $P_{\Delta \mu} \geq$ $1500 \mathrm{~d}$ for $\Delta \mu$ binaries (see Figs. 3 and 4). Let $P_{\mathrm{SB}}$ be the maximum period of spectroscopic binaries detectable with the radialvelocity coverage of the Geneva-Copenhagen catalogue, to be fixed later on. The relative fractions of those binaries may be computed from the period distribution of binaries, which has been shown to be $\log$ - normal (Duquennoy \& Mayor 1991; Halbwachs et al. 2003; Eggenberger et al. 2004a):

$f(\log P)=\frac{1}{s \sqrt{2 \pi}} \mathrm{e}^{-(1 / 2) z^{2}}$,

where $z=(\log P-4.8) / s$ and $s=2.3$, periods being expressed in days. More precisely, the fraction of binaries with periods in the range $P_{\mathrm{PM}}(=1000 \mathrm{~d})-P_{\mathrm{SB}}$ among binaries with periods $P<P_{\mathrm{SB}}$ may be expressed as $1-\Phi\left(z_{\mathrm{PM}}\right) / \Phi\left(z_{\mathrm{SB}}\right)$, where

$\Phi(z)=\frac{1}{\sqrt{2 \pi}} \int_{-\infty}^{z} \exp \left(\frac{-x^{2}}{2}\right) \mathrm{d} x$.

To obtain the computed fractions $1-\Phi\left(z_{\mathrm{PM}}\right) / \Phi\left(z_{\mathrm{SB}}\right)=0.36$ and $1-\Phi\left(z_{\Delta \mu}\right) / \Phi\left(z_{\mathrm{SB}}\right)=0.29$ consistent with the observed fractions $(0.357 \pm 0.025$ and $0.299 \pm 0.024$, respectively), it is required that $z_{\mathrm{SB}}=-0.42$ (or $P_{\mathrm{SB}}=6800 \mathrm{~d}$ ). The capability of the Geneva-Copenhagen survey to detect spectroscopic binaries up to periods of about $6800 \mathrm{~d}$ is not inconsistent with its typical radial-velocity sampling (see for instance Fig. 9).

We now evaluate the total number of proper-motion and spectroscopic binaries in the Geneva-Copenhagen catalogue. In

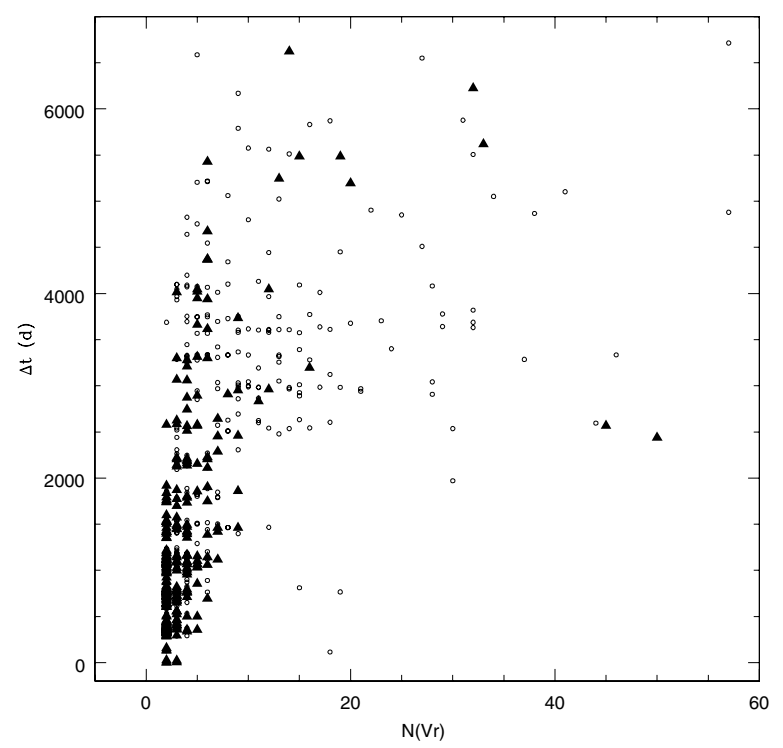

Fig. 9. Properties of the radial-velocity data for stars detected as propermotion binaries at the 0.9999 level: $N\left(V_{\mathrm{r}}\right)$ is the number of radialvelocity data points and $\Delta t$ their time span (in days). Open circles correspond to systems detected as spectroscopic binaries (SB) at the level $\operatorname{Prob}\left(\chi_{V_{\mathrm{r}}}^{2}\right) \leq 0.001$, and filled triangles to proper-motion binaries with constant radial velocity at the level $\operatorname{Prob}\left(\chi_{V_{\mathrm{r}}}^{2}\right)>0.001$. It is clear that most of the proper-motion binaries not detected as spectroscopic binaries have either radial-velocity data points that are scarce $\left(N\left(V_{\mathrm{r}}\right)<5\right)$, or that have a short time $\operatorname{span}(\Delta t<2000 \mathrm{~d})$.

total, 1892 stars out of 9431 with HIP and Tycho-2 entries in the Geneva-Copenhagen catalogue have $\operatorname{Prob}\left(\chi_{V_{\mathrm{r}}}^{2}\right) \leq 0.001$ and may thus be considered as bona fide spectroscopic binaries. On top of these, 384 stars are DMSA/G or $\Delta \mu$ binaries (at the 0.9999 level) not seen as spectroscopic binaries $\left(\operatorname{Prob}\left(\chi_{V_{\mathrm{s}}}^{2}\right)>0.001\right)$, for a total of 803 DMSA/G or propermotion binaries in the Geneva-Copenhagen catalogue (or 8.5\%). In total, the fraction of (spectroscopic + astrometric) binaries in the Geneva-Copenhagen survey thus amounts to $24.1 \pm 0.4 \%$ $[=(1892+384) / 9431]$. When restricting the sample to stars with parallaxes in excess of 20 mas, this frequency becomes $20.7 \pm$ $0.8 \%[=(364+131) / 2386]$.

The 384 proper-motion binaries not detected as spectroscopic binaries generally have few radial-velocity points $(<5)$ not covering a long time $\operatorname{span}(\Delta t<2000 \mathrm{~d})$, as can be seen in Fig. 9. However, thirty systems do not fall in this category: their radial-velocity data had the required properties to confirm their binary nature inferred from the proper-motion data, and yet they are not flagged as spectroscopic binaries at the level $\operatorname{Prob}\left(\chi_{V_{\mathrm{r}}}^{2}\right) \leq 0.001$ in the Geneva-Copenhagen survey. These systems are listed in Table 6 , under three different categories: (i) single stars spuriously flagged as proper-motion binaries because they exhibit perspective accelerations; (ii) confirmed spectroscopic or visual binaries; or (iii) stars suspected of being long-period binaries (and seen nearly pole-on, or with low-mass companions, or with very eccentric orbits). We discuss each category in turn in the remainder of this section.

The first category corresponds to a purely geometrical effect, caused by the perspective diminishing (increasing) the proper motion of a close, fastly receding (respectively approaching) object. Writing the transverse spatial velocity as $V_{\mathrm{t}}=\mu d=$ $|V| \cos \beta$ and the radial velocity as $V_{\mathrm{r}}=|V| \sin \beta$ (where $d$ is the distance to an object, $|V|=\left(V_{\mathrm{t}}^{2}+V_{\mathrm{r}}^{2}\right)^{1 / 2}$ is the modulus of the spatial velocity, $\beta$ is the angle between the tangential and spatial 
Table 6. Stars flagged as DMSA/G or (candidate) $\Delta \mu$-binaries, not detected as spectroscopic binaries in the Geneva-Copenhagen survey despite enough radial-velocity data points $\left(N\left(V_{\mathrm{r}}\right)>5\right)$ spanning more than $2000 \mathrm{~d}$. For spectroscopic binaries detected elsewhere, $P_{\mathrm{SB}}$ is the spectroscopic period, if applicable. If $K \mu$ [mas $\left.\mathrm{yr}^{-1}\right]\left|V_{\mathrm{r}}\right|\left[\mathrm{km} \mathrm{s}^{-1}\right] \varpi[\mathrm{mas}]>3.0 \times 10^{-2} \mathrm{mas} \mathrm{yr}^{-2}$, where $K=10^{-9}\left[\mathrm{kpc} \mathrm{km}^{-1} \mathrm{~s} \mathrm{yr}^{-1}\right]$ is a unit conversion factor to get $\dot{\mu}$ in mas $\mathrm{yr}^{-2}$, perspective acceleration is detectable.

\begin{tabular}{|c|c|c|c|c|c|c|c|}
\hline HIP & Name & $N\left(V_{\mathrm{r}}\right)$ & $\begin{array}{l}\Delta t\left(V_{\mathrm{r}}\right) \\
(\mathrm{d})\end{array}$ & $\operatorname{Prob}\left(\chi^{2}\left(V_{\mathrm{r}}\right)\right)$ & $\begin{array}{l}K \mu_{\mathrm{HIP}}\left|V_{\mathrm{r}}\right| \varpi \\
\left(\operatorname{mas~yr}^{-2}\right)\end{array}$ & $\begin{array}{l}P_{\mathrm{SB}} \\
(\mathrm{d})\end{array}$ & Remark \\
\hline \multicolumn{8}{|c|}{ Perspective acceleration } \\
\hline 24186 & Kapteyn & 6 & 4368 & 0.195 & 0.54 & - & \\
\hline 54035 & HD 95735 & 19 & 5487 & 0.081 & 0.16 & - & \\
\hline 99461 & HD 191408 & 9 & 2459 & 0.939 & 0.035 & - & \\
\hline 104217 & 61 Cyg B & 32 & 6226 & 0.723 & 0.094 & - & \\
\hline \multicolumn{8}{|c|}{ Binary systems } \\
\hline 12421 & HD 16619 & 6 & 3940 & 0.280 & $1 \times 10^{-4}$ & .. & visual binary $\left(0.1^{\prime \prime}\right)$ \\
\hline 29295 & HD 42581 & 15 & 5488 & 0.003 & $5 \times 10^{-4}$ & .. & $\begin{array}{l}\text { Gl } 229 \text { B brown dwarf } \\
\text { (Nakajima et al. 1995) }\end{array}$ \\
\hline 29860 & HD 43587 & 9 & 2953 & 0.010 & $1 \times 10^{-4}$ & 12309 & $\begin{array}{l}M_{\text {comp }} \geq 0.34 M_{\odot} \text { (Vogt et al. } \\
2002)\end{array}$ \\
\hline 60268 & HD 107582 & 6 & 4676 & 0.805 & $8 \times 10^{-4}$ & 6864 & SB (Latham et al. 2002) \\
\hline 67275 & $\tau$ Boo & 33 & 5618 & 0.002 & $5 \times 10^{-4}$ & $3.3+$. & $\begin{array}{l}\text { exoplanet in a wide bi- } \\
\text { nary (Patience et al. 2002; } \\
\text { Eggenberger et al. 2004b) }\end{array}$ \\
\hline 80902 & HD 150706 & 12 & 4048 & 0.670 & $8 \times 10^{-5}$ & 264 & $\begin{array}{l}\text { exoplanetary companion (Udry } \\
\text { et al. 2003) }\end{array}$ \\
\hline 102431 & HD 198084 & 14 & 6625 & 0.036 & $3 \times 10^{-4}$ & 523 & SB2 (Griffin 1999) \\
\hline 110618 & $v$ Ind & 16 & 3196 & 0.612 & $1 \times 10^{-3}$ &.. & $\begin{array}{l}\text { visual binary }\left(0.1^{\prime \prime}\right) \text { : A3V? + } \\
\text { F9 }\end{array}$ \\
\hline \multicolumn{8}{|c|}{ Candidate binaries with low-mass companions (or nearly pole-on systems or very eccentric systems) } \\
\hline 493 & HD 101 & 7 & 2452 & 0.924 & $2 \times 10^{-4}$ & - & \\
\hline 4446 & HD 5357 & 6 & 4375 & 0.128 & $1 \times 10^{-5}$ & - & \\
\hline 8674 & HD 11397 & 6 & 2206 & 0.923 & $2 \times 10^{-4}$ & - & $\begin{array}{l}\text { CH subdwarf (Šleivyte \& } \\
\text { Bartkevičius 1990) }\end{array}$ \\
\hline 16788 & HD 22309 & 13 & 5247 & 0.045 & $2 \times 10^{-4}$ & - & non SB in Latham et al. (2002) \\
\hline 19335 & HD 25998 & 8 & 2908 & 0.234 & $3 \times 10^{-4}$ & - & $\begin{array}{l}\text { no low-mass companion de- } \\
\text { tected with ELODIE (Galland } \\
\text { et al. 2005) }\end{array}$ \\
\hline 44896 & HD 78612 & 45 & 2567 & 0.008 & $8 \times 10^{-4}$ & - & \\
\hline 65268 & HD 116316 & 7 & 2288 & 0.571 & $8 \times 10^{-5}$ & - & \\
\hline 66319 & HD 118186 & 50 & 2438 & 0.226 & $8 \times 10^{-6}$ & - & \\
\hline 91215 & HD 171706 & 6 & 2113 & 0.685 & $5 \times 10^{-5}$ & - & \\
\hline 95703 & HD 183473 & 6 & 3615 & 0.003 & $8 \times 10^{-5}$ & - & \\
\hline 98192 & HD 189087 & 20 & 5194 & 0.980 & $3 \times 10^{-4}$ & - & \\
\hline 101726 & HD 196141 & 6 & 3299 & 0.085 & $8 \times 10^{-5}$ & - & \\
\hline 103260 & HD 198829 & 9 & 3734 & 0.015 & $1 \times 10^{-5}$ & - & \\
\hline 103859 & HD 200560 & 12 & 2961 & 0.918 & $3 \times 10^{-4}$ & - & \\
\hline 108095 & HD 208068 & 7 & 2644 & 0.932 & $9 \times 10^{-5}$ & - & \\
\hline 109461 & LHS 3767 & 11 & 2835 & 0.015 & $4 \times 10^{-4}$ & - & \\
\hline 111004 & HD 213122 & 6 & 2227 & 0.111 & $2 \times 10^{-7}$ & - & \\
\hline 112117 & HD 214953 & 6 & 5429 & 0.714 & $2 \times 10^{-4}$ & - & \\
\hline
\end{tabular}

velocities, such that $\dot{\beta}=\mu$ ) and expressing that, for a single star, $|V|$ remains constant over time, yields

$\dot{\mu}=-2 \mu V_{\mathrm{r}} d^{-1}$.

This perspective acceleration $\dot{\mu}$ may be detected as a secular change of the proper motion, and confused with a proper-motion binary. The variation $\Delta \mu$ of the proper motion over the interval $\Delta t$ may be written as:

$|\Delta \mu|=\left|2 \mu V_{\mathrm{r}} d^{-1} \Delta t\left(1-V_{\mathrm{r}} d^{-1} \Delta t\right)\right|$,

corresponding to the second-order Taylor expansion of the solution of Eq. (12). Perspective acceleration should be detectable by our $\chi^{2}$ test with a threshold of 18.4206 for stars having $|\Delta \mu|>4.3 \sigma_{\Delta \mu}$, where $\sigma_{\Delta \mu}$ is a typical error of $\alpha$ or $\delta$ component of the proper motion difference between Hipparcos and Tycho-2.
In practice, the situation is a bit more complicated, since "instantaneous" proper motions (as those appearing in the previous formula) cannot be determined. Proper motions are always computed from differences of positions extending over some time interval, and the effect of perspective acceleration on the positions is proportional to $\dot{\mu} \Delta t^{2}$. The Tycho-2 proper motion is derived by a linear least-square fit on these positions spanning $\Delta t \sim 100$ yr: the Tycho-2 proper motion will therefore fall somewhere in the range defined by the "instantaneous" proper motions corresponding to each end of the time interval $\Delta t$, so that $\left|\mu_{\mathrm{HIP}}-\mu_{\text {Tycho-2 }}\right|<|\Delta \mu|$, with $|\Delta \mu|$ expressed by Eq. (13).

There is no need here, however, to go beyond the simple approximation expressed by the inequality $\left|2 \mu V_{\mathrm{r}} d^{-1} \Delta t\right|>$ $4.3 \sigma_{\Delta \mu}$ (with $\Delta t \sim 100 \mathrm{yr}$ and $\sigma_{\Delta \mu} \sim 1.4 \mathrm{mas} \mathrm{y}^{-1}$, as suitable for Hipparcos and Tycho-2 measurements), since the purpose of 
the whole discussion is just to identify stars showing perspective accelerations mistaken for $\Delta \mu$ binaries. These stars are listed in Table 6 (and were already identified as perspective-acceleration stars in Table 1.2.3 of Volume 1 of the Hipparcos and Tycho Catalogues; ESA 1997). For none of the stars listed in Table 6 is there a need to go beyond the second-order Taylor expansion considered in Eq. (13).

All but four stars in Table 6 do not, by far, fulfill the criterion for perspective acceleration. An extensive literature search for these 26 stars resulted in 8 stars with clear indications for being close visual binaries or spectroscopic binaries. Interestingly enough, two among these 8 systems have low-mass (HIP 29860; Vogt et al. 2002) or even brown-dwarf companions (HIP 29295 = Gliese 229; Nakajima et al. 1995). They constitute further examples of the ability of the $\Delta \mu$ method to detect binaries with low-mass companions, adding to the case of HIP 25647 already discussed by Makarov \& Kaplan (2005). In the case of HIP 80902 (=HD 150706), which has been announced to host a 1 Jupiter-mass exoplanet (Udry et al. 2003), one may however doubt that the $\Delta \mu$ method is able to detect companions that light (see the discussion of Makarov \& Kaplan 2005, in relation with their Eq. (2)). The 18 stars in Table 6 neither exhibiting perspective accelerations nor already known as binaries are flagged candidate binaries with possibly low-mass companions, although very low inclinations or very eccentric orbits could be other explanations for their non-detection as spectroscopic binaries by the Geneva-Copenhagen survey.

\section{4. $K$ and $M$ giants in the solar neighbourhood}

Famaey et al. (2005) published a study of the local kinematics of $\mathrm{K}$ and $\mathrm{M}$ giants, accompanied by a catalogue that contains 6691 such objects, possessing Hipparcos and Tycho-2 proper motions, as well as CORAVEL radial velocities. The sample can thus be subject to a similar analysis as done above for the Geneva-Copenhagen catalogue. Again, a threshold of 0.999 on 1-Prob $\left(\chi_{V_{\mathrm{r}}}^{2}\right)$ to identify stars with variable radial velocities has been adopted. The confrontation of spectroscopic and $\Delta \mu$ binaries must, however, be restricted to the $5680 \mathrm{~K}$ giants, since in $\mathrm{M}$ giants intrinsic radial-velocity variations (related to envelope pulsations) make the $\operatorname{Prob}\left(\chi_{V_{\mathrm{r}}}^{2}\right)$ criterion useless to identify spectroscopic binaries (see Fig. 5 of Famaey et al. 2005).

This section presents an analysis similar to that performed for the Geneva-Copenhagen sample of F-G dwarfs (Sect. 5.3; compare Figs. 8 and 10) for $\mathrm{K}$ giants. The fraction of $\Delta \mu$ binaries (at the confidence level of 0.9999) among spectroscopic binaries $\left[\operatorname{Prob}\left(\chi_{V_{\mathrm{r}}}^{2}\right) \leq 0.001\right]$ amounts to $5.6 \%(=46 / 816)$, raising to $21.8 \%$ when adding the DMSA/G systems. These fractions both become equal to $53.8 \pm 13.8 \%(=7 / 13)$ when eliminating those stars with parallaxes smaller than 20 mas among which proper-motion binaries are not efficiently detected. The latter frequency differs by about $1.5 \sigma$ from the corresponding values for the Geneva-Copenhagen sample, and a possible cause for that difference will be suggested in Sect. 6. The large errors on the K-giant frequencies reflect the fact that they are derived on a small sample, since not many $\mathrm{K}$ giants have parallaxes in excess of 20 mas.

We now evaluate the total number of proper-motion and spectroscopic binaries among $\mathrm{K}$ giants. In total, 816 stars out of 5680 have $\operatorname{Prob}\left(\chi_{V_{\mathrm{r}}}^{2}\right) \leq 0.001$ and may thus be considered as bona fide spectroscopic binaries. On top of these, 82 stars are DMSA/G or $\Delta \mu$ binaries (at the 0.9999 level) not seen as spectroscopic binaries $\left(\operatorname{Prob}\left(\chi_{V_{V}}^{2}\right)>0.001\right)$, for a total of $178 \mathrm{DMSA} / \mathrm{G}$ or proper-motion binaries. The fraction of

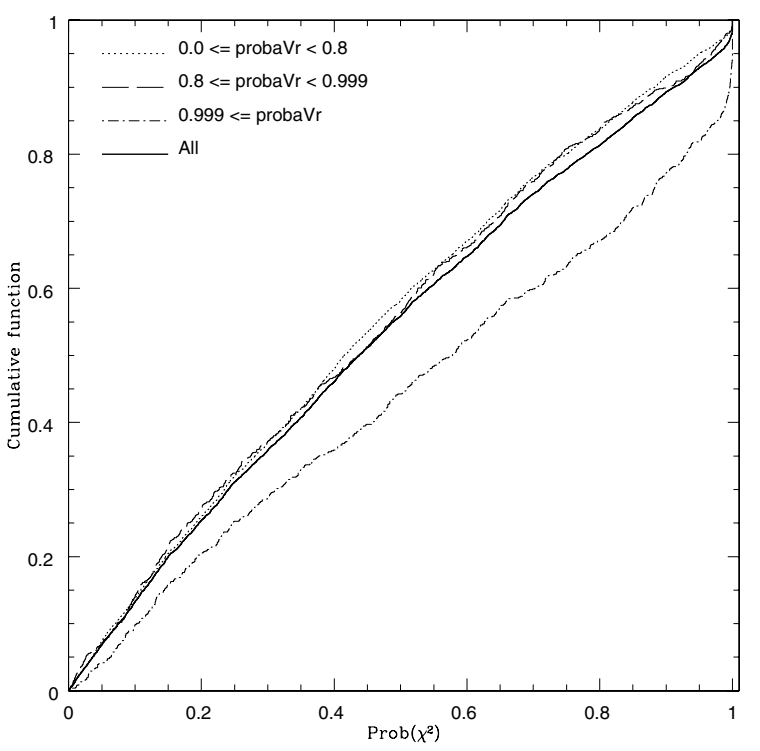

Fig. 10. Same as Fig. 8, but for the K giants from the sample of Famaey et al. (2005). The largest fraction of proper-motion binaries is indeed found among radial-velocity binaries, having probaVr $\geq 0.999$.

(spectroscopic + astrometric) binaries among $\mathrm{K}$ giants thus amounts to $15.8 \%[=(816+82) / 5680]$. This fraction raises to $30.2 \pm 6.3 \%[=(13+3) / 53]$ when restricting the sample to $\mathrm{K}$ giants with parallaxes in excess of 20 mas. This frequency again differs by $1.5 \sigma$ from the corresponding $20.7 \pm 0.8 \%$ frequency derived for the Geneva-Copenhagen sample.

Among the 82 proper-motion binaries not detected as spectroscopic binaries, only two (HIP 24450 and HIP 78159) have more than 5 radial-velocity measurements spanning more than $1000 \mathrm{~d}$, thus with the potential to detect a possible long-period orbit. Actually, HIP 78159 is a visual binary with $1.8^{\prime \prime}$ angular separation and a period of 41.56 y (about $15000 \mathrm{~d}$; see Dvorak et al. 1989, and references therein) which most probably corresponds to the proper-motion binary. No independent confirmation is available in the literature about the binary nature of HIP 24450.

\section{Fraction of proper-motion binaries as a function of spectral type}

Unbiased estimates of the proportion of binaries among different spectral types are rare, so that the opportunity offered by the $\Delta \mu$ and DMSA/G binaries is worth investigating. The efficiency of detecting $\Delta \mu$ binaries is affected by the distance to a tested object. Among farther objects more will be missed by the method due their smaller (angular) proper motions. To obtain a reliable estimate of the binary fraction, it is necessary to restrict the sample to the distance (i.e., parallax) region where the $\Delta \mu$ method attains its full efficiency. Figure 11 displays the detection fraction as a function of parallax for $G$ type stars in our complete sample. It can be seen that a plateau in the detected binary fraction is reached beyond 20 mas. In the following analysis of the $\Delta \mu$ binary frequency among the various spectral types, the sample has therefore been restricted to stars with parallaxes larger than 20 mas.

Table 7 lists the fraction of proper-motion binaries among the various spectral types. Because $\Delta \mu$ and DMSA/G binaries probe slightly different period ranges $(\Delta \mu$ binaries not flagged as DMSA/G, as listed in Col. 5, represent the long-period tail 
Table 7. Fraction of proper-motion binaries of a given type among various spectral classes, normalised by the total number of stars in the considered spectral class (labelled $N_{\text {tot }}$ ). Uncertainties on the listed frequencies are derived from the binomial distribution. The large fluctuations observed in Col. (1) are not physical and are caused by the different parallax distributions among the different spectral types (see Col. (6) and text).

\begin{tabular}{|c|c|c|c|c|c|c|c|c|}
\hline Spectral type & $N_{\text {tot }}$ & (1) & (2) & (3) & (4) & (5) & (6) & Catalogue \\
\hline WR-O & 251 & $0.012 \pm 0.007$ & $<0.004$ & $<0.004$ & $<0.004$ & $<0.004$ & $0.4 \%$ & \\
\hline B & 8664 & $0.022 \pm 0.001$ & $0.143 \pm 0.132$ & $0.143 \pm 0.132$ & $0.143 \pm 0.132$ & $<0.0001$ & $0.8 \%$ & \\
\hline A & 15662 & $0.033 \pm 0.001$ & $0.152 \pm 0.030$ & $0.131 \pm 0.028$ & $0.083 \pm 0.022$ & $0.069 \pm 0.021$ & $0.9 \%$ & \\
\hline $\mathrm{F}$ & 21340 & $0.046 \pm 0.001$ & $0.114 \pm 0.010$ & $0.091 \pm 0.009$ & $0.063 \pm 0.008$ & $0.051 \pm 0.007$ & $4.3 \%$ & \\
\hline G & 19628 & $0.043 \pm 0.001$ & $0.105 \pm 0.008$ & $0.091 \pm 0.007$ & $0.052 \pm 0.006$ & $0.053 \pm 0.006$ & $8.2 \%$ & \\
\hline $\mathrm{K}$ & 29349 & $0.026 \pm 0.001$ & $0.093 \pm 0.007$ & $0.070 \pm 0.006$ & $0.051 \pm 0.005$ & $0.042 \pm 0.005$ & $6.2 \%$ & \\
\hline M & 4217 & $0.029 \pm 0.002$ & $0.098 \pm 0.013$ & $0.077 \pm 0.012$ & $0.036 \pm 0.008$ & $0.061 \pm 0.010$ & $12.3 \%$ & \\
\hline All & 103304 & $0.0346 \pm 0.0005$ & $0.101 \pm 0.004$ & $0.080 \pm 0.004$ & $0.053 \pm 0.003$ & $0.048 \pm 0.003$ & $5.3 \%$ & \\
\hline$\overline{\mathrm{Am}}$ & 1356 & $0.075 \pm 0.007$ & $0.167 \pm 0.048$ & $0.133 \pm 0.044$ & $0.117 \pm 0.041$ & $0.050 \pm 0.028$ & $4.4 \%$ & (a) \\
\hline Gen-Cop (F-G V) & 9431 & $0.085 \pm 0.003$ & $0.109 \pm 0.006$ & $0.089 \pm 0.006$ & $0.057 \pm 0.005$ & $0.052 \pm 0.004$ & $25.3 \%$ & (b) \\
\hline Gliese (G-K V) & 236 & $0.106 \pm 0.020$ & $0.109 \pm 0.021$ & $0.087 \pm 0.018$ & $0.043 \pm 0.013$ & $0.065 \pm 0.016$ & $97.5 \%$ & (c) \\
\hline K III & 5680 & $0.031 \pm 0.002$ & $0.189 \pm 0.054$ & $0.189 \pm 0.054$ & $0.132 \pm 0.046$ & $0.057 \pm 0.031$ & $0.9 \%$ & (d) \\
\hline \multirow[t]{2}{*}{$\mathrm{Ba}$} & 156 & $0.090 \pm 0.023$ & $0.27 \pm 0.07^{*}$ & $0.19 \pm 0.06^{*}$ & $0.16 \pm 0.06^{*}$ & $0.11 \pm 0.05^{*}$ & $23.7 \% *$ & (e) \\
\hline & & & $0.50 \pm 0.14^{* *}$ & $0.50 \pm 0.14^{* *}$ & $0.17 \pm 0.11^{* *}$ & $0.33 \pm 0.14^{* *}$ & $7.7 \%^{* *}$ & (e) \\
\hline
\end{tabular}

Column headings:

(1) $\Delta \mu\left(\operatorname{Prob}\left(\chi^{2}\right)>0.9999\right)+\mathrm{DMSA} / \mathrm{G}($ All Prob), all $\varpi$.

(2) $\Delta \mu\left(\operatorname{Prob}\left(\chi^{2}\right)>0.9999\right)+$ DMSA/G (All Prob), $\varpi>20$ mas.

(3) $\Delta \mu\left(\operatorname{Prob}\left(\chi^{2}\right)>0.9999\right), \varpi>20$ mas.

(4) DMSA/G, All Prob $\left(\chi^{2}\right), \varpi>20$ mas.

(5) Only $\Delta \mu\left(\operatorname{Prob}\left(\chi^{2}\right)>0.9999\right)$, no DMSA/G (All Prob), $\varpi>20$ mas.

(6) Fraction of stars in the considered spectral class with $\varpi>20$ mas.

${ }^{*}$ For barium stars, these frequencies refer to a parallax threshold of 5 instead of 20 mas.

** For barium stars, these frequencies refer to a parallax threshold of 10 instead of 20 mas. Catalogue reference:

(a) Renson et al. (1991); (b) Nordström et al. (2004); (c) Halbwachs et al. (2003); (d) Famaey et al. (2005); (e) Lü et al. (1983).

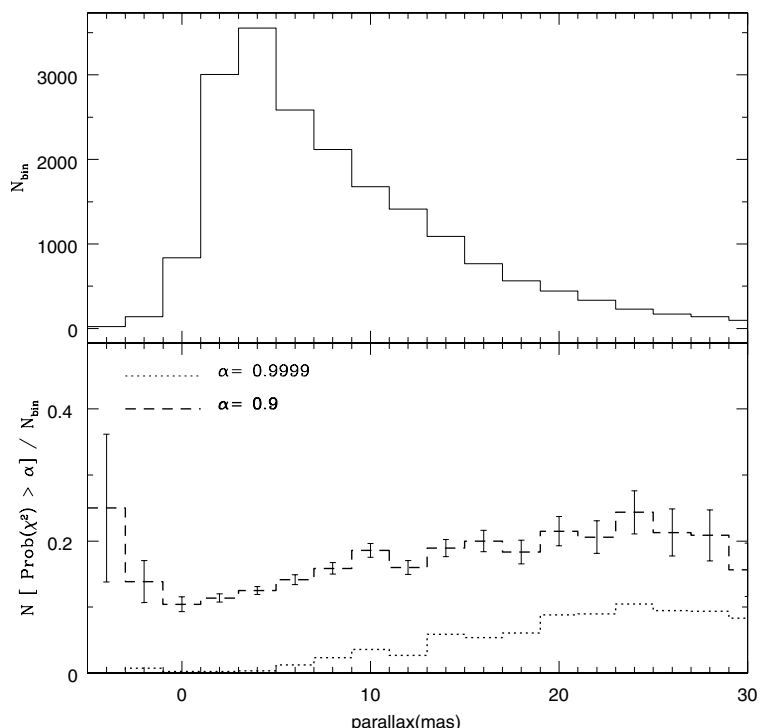

Fig. 11. Upper panel: the parallax distribution of stars with spectral type $\mathrm{G}$ in the Hipparcos catalogue. Lower panel: fraction of stars detected as $\Delta \mu$ binaries (at confidence levels 0.9 - dashed line - and 0.9999 - dotted line) in a given parallax bin. Note how the dotted curve reaches its final level of $10 \%$ for parallaxes larger than 20 mas. At 10 mas, only half of the expected systems are detected.

among proper-motion binaries: see Fig. 4), they have been listed separately in Table 7 . The importance of restricting the sample to stars with parallaxes larger than 20 mas is very clearly illustrated by the comparison of Cols. 1 and 2. The fraction of proper-motion binaries stays basically constant (within the error bars) among the various spectral types (Cols. 2 to 5) when restricting the sample to parallaxes larger than 20 mas, in contrast to the situation prevailing for the full sample (Col. 1). Since the proportion of stars with $\varpi>20$ mas (Col. 6) depends upon the spectral type (because stars of different spectral types - and hence average luminosities - have different average distances in a magnitude-limited sample), the fraction of the sample in which proper-motion binaries are efficiently detected varies with spectral type, resulting in the variations observed in Col. 1 of Table 7. Since this selection bias was not properly recognised by Makarov \& Kaplan (2005), these authors concluded that the fraction of long-period binaries is larger among solar-type stars than among the other spectral classes (as is indeed suggested by Col. 1 of Table 7). Based on the above discussion, this conclusion must be discarded, however, as shown by the nearly constant frequencies listed in Cols. 2 to 5 of Table 7. For instance, the fraction of proper-motion binaries among F-G stars is now about the same for the Geneva-Copenhagen sample, for the whole Hipparcos Catalogue and for $\mathrm{G}$ and $\mathrm{K}$ dwarfs from the Gliese catalogue (Halbwachs et al. 2003).

If anything, the fraction of proper-motion binaries seems (slightly) larger among spectral types associated with high luminosities, like the upper main sequence (B and A types) and the $\mathrm{K}$ giants. This effect may again result from a selection bias, remembering that proper-motion binaries cannot be detected in binaries with components of nearly equal luminosities (since in that case, the photocentre will be located close to the centre of mass, and there will not be any detectable orbital motion): in binaries with a high-luminosity primary star, there is a larger luminosity range accessible for the secondary (belonging to the lower main sequence), thus increasing the probability of detecting those systems as proper-motion binaries.

In the same vein, it should be remarked in Fig. 11 that there is a large fraction of $\Delta \mu$ binaries among stars with negative parallaxes (at least when the confidence level is relaxed from 0.9999 to 0.9 ), suggesting that the same disturbing effect (unrecognised orbital motion?) that pushed the astrometric solution into 
Table 8. Candidate $\Delta \mu$ binaries at the 0.9999 confidence level or DMSA/G binaries among long-period variable stars. The column labelled $\varpi($ rev) lists the parallax recomputed as explained in Knapp et al. (2003). See text for a word of caution about the reality of these candidate binaries, inspired by their very small parallaxes.

\begin{tabular}{lllrcrc}
\hline \hline HIP & GCVS & Prob $\left(\chi^{2}\right)$ & $\chi^{2}$ & DMSA & $\begin{array}{r}\varpi(\mathrm{HIP}) \\
(\mathrm{mas})\end{array}$ & $\begin{array}{c}\varpi(\mathrm{rev}) \\
(\mathrm{mas})\end{array}$ \\
\hline 10826 & o Ceti & 1.0000000 & 48.66 & 5 & 7.79 & $9.34 \pm 1.07$ \\
14042 & T Hor & 0.9999998 & 30.94 & $\mathrm{~V}$ & -0.15 & $0.69 \pm 1.22$ \\
28874 & S Lep & 0.9999836 & 22.03 & 5 & 3.63 & - \\
34859 & VX Gem & 1.0000000 & 46.72 & 5 & 1.05 & $1.02 \pm 1.99$ \\
78872 & Z Sco & 0.1404664 & 0.30 & $\mathrm{G}$ & -2.47 & $1.71 \pm 2.56$ \\
84948 & RS Her & 0.9309712 & 5.34 & $\mathrm{G}$ & 1.10 & $0.80 \pm 1.41$ \\
85617 & TW Oph & 1.0000000 & 37.73 & $\mathrm{~V}$ & 3.57 & $3.66 \pm 1.17$ \\
\hline
\end{tabular}

unphysical territory may have led to (slightly) incorrect Hipparcos proper motions. Finally, it is worth nothing that, for positive parallaxes, the fraction of $\Delta \mu$ binaries at the 0.9 confidence level runs almost parallel to the curve corresponding to 0.9999 , the two curves being offset by $10 \%$, corresponding to the fraction of false detections expected at the 0.9 confidence level. This constitutes a further confirmation of the validity of the statistical treatment described in Sect. 2.

Barium stars are an interesting comparison sample since they are known to be all binaries (see the discussion in Sect. 5.2), and yet the frequency of $\Delta \mu$ binaries listed in Col. (1) of Table 7 is only $0.09 \pm 0.02$ because of the small proportion of barium stars with large parallaxes (only 2 stars have parallaxes larger than 20 mas). To get meaningful frequencies, it has thus been necessary to lower the parallax threshold adopted for barium stars in Table 7 (see also Sect. 5.2). Two different values have been adopted: 5 mas, to ensure consistency with the discussion of Sect. 5.2, and 10 mas to get a somewhat larger detection efficiency of proper-motion binaries. Although significantly larger - as expected - than the frequency of binaries found in the other spectral classes, this frequency is not unity because (i) the 5 and 10 mas parallax thresholds only ensure a partial (about 30 to 50\%; see Figs. 5, 7, and 11) detection of the propermotion binaries; (ii) as already discussed in Sect. 5.3, $\Delta \mu$ binaries are restricted to a limited range in orbital periods (about 1500-30000 d, as clearly apparent from Fig. 4), whereas barium binaries extend beyond that period range.

Am stars are another interesting class to evaluate the frequency of $\Delta \mu$ binaries, in the framework of a possible relationship between this class and the barium stars (Hakkila 1989; Jorissen \& Van Eck 2005). The arguments in support of such a connection are based on the binary nature of stars from both classes, on the similarities of their abundance peculiarities and of their kinematical properties. The main argument to reject any such evolutionary link, however, is based on the significantly different period ranges observed among Am and barium stars. However, the question arises whether long-period binaries are really lacking among Am stars, or whether this lack is a consequence of the difficulty of identifying them from a radialvelocity monitoring. It is possible to check this alternative by deriving the fraction of $\Delta \mu$ binaries among the sample of $1356 \mathrm{Am}$ (and Ap) stars from the catalogue of Renson et al. (1991) having an HIP entry. That fraction is the same as for A-F dwarfs so that there is no hint for more long-period binaries among Am stars than among the comparison sample.

$\mathrm{S}$ stars without lines from the unstable element Tc (also called extrinsic S stars; Van Eck et al. 1998) are a family of binary stars closely related to barium stars (Jorissen et al. 1998; Jorissen 2003). It is therefore of interest to check the fraction of
$\Delta \mu$ binaries among them as well. The situation is, however, less favourable for extrinsic $S$ stars than for barium stars because (i) extrinsic S stars are more luminous (hence they are more distant on average and thus have smaller parallaxes) than barium stars (Van Eck et al. 1998); and (ii) they are mixed with intrinsic $\mathrm{S}$ stars (i.e., genuine asymptotic giant branch stars), which need not be binaries. Actually, none among the extrinsic S stars in the Hipparcos Catalogue has a parallax in excess of 10 mas, and it is therefore not surprising that no $\Delta \mu$ binary is present among them. The only $\Delta \mu$ binary detected among S stars is HIP 110487 ( $\left.\pi^{1} \mathrm{Gru}\right)$, an intrinsic $S$ star that is known to be a visual binary with a G0V companion 2.7" away (Proust et al. 1981), for which an orbital period of $6000 \mathrm{yr}$ is inferred (Knapp et al. 1999). With such a long orbital period, one may actually wonder whether the visual companion is really the one responsible for the $\Delta \mu$ binary, or whether there could be another, closer companion that plays some role as well in the shaping of the complex circumstellar environment inferred from the submm CO lines by Knapp et al. (1999), Chiu et al. (2006), and Cruzalèbes \& Sacuto (2006).

The search for $\Delta \mu$ binaries among long-period variables (LPVs) offers an interesting alternative to radial-velocity monitoring since spectroscopic binaries are very difficult to detect among LPVs, due to the intrinsic radial-velocity jitter caused by their pulsation (Jorissen 2003). Table 8 reveals a few $\Delta \mu$ and DMSA/G binaries among the 233 LPVs from the Hipparcos Catalogue collected by Knapp et al. (2003). These candidate proper-motion binaries are unusual in the sense that they are detected among stars with very low parallaxes. In fact, among LPVs with parallaxes in excess of 10 mas (only 3 stars), there are no candidate binaries. This casts doubts on the reality of the binary detection for stars with parallaxes as small as those listed in Table 8. Except for o Cet, there is unfortunately no independent confirmation of their duplicity. Parallaxes of very red stars such as LPVs are subject to specific instrumental effects like chromaticity, causing a displacement of their stellar image on the detector (as compared to the image location of a bluer star occupying the same position on the sky), as discussed by Knapp et al. (2003) and Platais et al. (2003). It is possible that this chromatic effect, if not properly corrected on the older plates used by the Tycho-2 consortium to derive the proper motion, could spoil its determination and produce spurious $\Delta \mu$ binaries.

Finally, to conclude this section, it is interesting to list the few candidate $\Delta \mu$ binaries found among Wolf-Rayet (WR) and O-type stars, adding to the spectroscopic and visual binaries listed by Mason et al. (1998) and Niemela et al. (1999). Individual properties of $\Delta \mu$ binaries among WR and $\mathrm{O}$ stars, down to a confidence level of $\operatorname{Prob}\left(\chi^{2}\right)>0.99$, are listed in Table 9. This list may be compared to that of Hartkopf et al. (1999) and Mason et al. (1998), who searched for visual 
Table 9. Candidate $\Delta \mu$ binaries at the 0.99 confidence level or DMSA/G binaries among Wolf-Rayet and O-type stars. The column labelled $\varpi$ lists the HIPPARCOS parallax. SB in column "remark" stands for "spectroscopic binary". See text for a word of caution about the reality of these candidate binaries, inspired by their very small parallaxes.

\begin{tabular}{lllrllrl}
\hline \hline HIP & HD & Prob $\left(\chi^{2}\right)$ & $\chi^{2}$ & DMSA & Sp. type & $\begin{array}{r}\varpi(\mathrm{HIP}) \\
(\mathrm{mas})\end{array}$ & Remark \\
\hline 48617 & 86161 & 0.9921 & 9.69 & 5 & WR 16 & $-1.01 \pm 0.74$ & $\mathrm{SB}^{a} 10.73 \mathrm{~d}$ \\
68995 & 123008 & 0.9963 & 11.21 & 5 & $\mathrm{O}$ & $1.95 \pm 1.30$ & \\
89769 & 168206 & 0.9989 & 13.62 & 5 & WR 113 & $1.38 \pm 1.32$ & $\mathrm{SB}^{b} 29.1 \mathrm{~d}$ \\
99768 & 192639 & 0.9926 & 9.83 & 5 & $\mathrm{O}$ & $1.22 \pm 0.64$ & \\
114685 & 219286 & 0.9994 & 14.98 & 5 & $\mathrm{O} 7$ & $2.04 \pm 0.90$ & \\
\hline
\end{tabular}

(a) Moffat \& Niemela (1982); (b) Massey \& Niemela (1981); Niemela et al. (1999).

binaries among $\mathrm{O}$ and WR stars using speckle interferometry. These speckle observations could not confirm the duplicity of any of the stars of Table 9. Here as for LPVs, the small parallaxes cast doubts on the reality of the candidate binaries.

\section{Conclusions}

The possibility of detecting binaries from the inconsistency between short-span (like Hipparcos) and long-span (like Tycho-2) proper motions (an idea put forward by Wielen 1997; Wielen et al. 1998; Makarov \& Kaplan 2005) has been investigated using test samples of known (spectroscopic) binaries. Using Hipparcos, Tycho-2, and $S_{\mathrm{B}^{9}}$ data, we show that almost all binaries in the period range 1000-30000 d (the lower bound corresponding to the span of the Hipparcos mission) are indeed detected as "proper-motion binaries". In the lower range 1000 to $2000 \mathrm{~d}$, binaries are best detected from their accelerated proper motions (flagged as DMSA/G in the Hipparcos Double and Multiple Systems Annex). Proper-motion binaries with $S_{\mathrm{B}^{9}}$ periods below $1000 \mathrm{~d}$ are good candidate triple stars, the propermotion binary involving a component with a longer orbital period than the $S_{\mathrm{B}^{9}}$ period. A detailed literature search indeed confirms the triple nature of 16 systems, and a list of 19 candidate triple systems is provided.

The comparison of short-span and long-span proper motions thus offers a promising way to detect binaries in extended stellar samples, and has a bright future given the various upcoming space astrometry missions like SIM and Gaia. Propermotion binaries also fill the gap between spectroscopic and speckle/interferometric binaries. When estimating the frequency of binaries in a given sample, their contribution is not negligible: in the Geneva-Copenhagen sample of F and G stars, propermotion binaries not detected as spectroscopic binaries add $5.5 \%$ to the fraction of spectroscopic binaries, $15.2 \%$, for a total of $20.7 \pm 0.8 \%$. In the Geneva-Copenhagen sample, there are several proper-motion binaries not detected as spectroscopic binaries, despite adequate radial-velocity coverage. These binaries may either be pole-on systems, very eccentric systems or systems with low-mass companions.

The frequency of proper-motion binaries in fact appears to be the same among all spectral classes (about $10.1 \pm 0.4 \%$ in the whole F-M range), contrasting with the claim of Makarov \& Kaplan (2005) that it is higher among solar-type stars (because these authors did not account for the selection biases). There is instead a hint that slightly more proper-motion binaries are observed for systems with luminous primaries (AV or KIII), as expected if the light ratio with their companion is generally larger than for systems hosting primaries on the lower main sequence. Hence, the photocentric motion is expected to be more frequently detectable - all other parameters being equal - for the former than for the latter.

As expected, barium stars exhibit a significantly larger fraction of proper-motion binaries $(50 \pm 15 \%$ for stars with $\varpi>$ 10 mas, consistent with $100 \%$ when duly accounting for the $\sim 50 \%$ detection efficiency of proper-motion binaries when $\varpi \sim$ 10 mas). There is no hint at a similarly large fraction of longperiod binaries among Am stars; the suspicion of a link between these two classes must thus be definitely abandoned. No convincing candidate proper-motion binaries emerged either among WR and $\mathrm{O}$ stars, or among long-period variable stars.

Acknowledgements. A.F. is a Foreign Postdoctoral Researcher from FNRS (Belgium) under the grant 1.5.108.05F. This research was supported in part by an ESA/PRODEX Research Grant (15152/01/NL/SFe).

\section{References}

Baranne, A., Mayor, M., \& Poncet, J. L. 1979, Vistas Astron., 23, 279

Boss, B., Albrecht, S., Jenkins, H., et al. 1937, General catalogue of 33342 stars for the epoch 1950, Washington, D.C., Carnegie Institution of Washington, Publ. No 486

Carquillat, J.-M., Pedoussaut, A., Ginestet, N., \& Nadal, R. 1976, A\&AS, 23, 277

Chiu, P.-J., Hoang, C.-T., Dinh-V-Trung, et al. 2006, ApJ, 645, 605

Cruzalèbes, P., \& Sacuto, S. 2006, A\&A, 452, 885

Duquennoy, A., \& Mayor, M. 1991, A\&A, 248, 485

Dvorak, R., Froeschle, C., \& Froeschle, C. 1989, A\&A, 226, 335

Eggenberger, A., Halbwachs, J.-L., Udry, S., \& Mayor, M. 2004a, Rev. Mex. Astron. Astrofis. Conf. Ser., 28

Eggenberger, A., Udry, S., \& Mayor, M. 2004b, A\&A, 417, 353

ESA 1997, The Hipparcos and Tycho Catalogues, ESA

Famaey, B., Jorissen, A., Luri, X., et al. 2005, A\&A, 430, 165

Fekel, F. C., Barlow, D. J., Scarfe, C. D., Jancart, S., \& Pourbaix, D. 2005, AJ, 129, 1001

Fekel, F. C., Barlow, D. J., Scarfe, C. D., \& Pourbaix, D. 2006, AJ, submitted Galland, F., Lagrange, A.-M., Udry, S., et al. 2005, A\&A, 444, L21

Goecking, K.-D., Duerbeck, H. W., Plewa, T., et al. 1994, A\&A, 289, 827

Gontcharov, G. A., \& Kiyaeva, O. V. 2002, A\&A, 391, 647

Griffin, R. F. 1999, The Observatory, 119, 272

Griffin, R. F., \& Cornell, A. P. 1997, The Observatory, 117, 82

Hakkila, J. 1989, AJ, 98, 699

Halbwachs, J. L., Mayor, M., Udry, S., \& Arenou, F. 2003, A\&A, 397, 159

Hartkopf, W., Mason, B., Wycoff, G., \& McAlister, H. 2001,

http: //ad.usno.navy.mil/wds/int4.html

Hartkopf, W. I., Mason, B. D., Gies, D. R., et al. 1999, AJ, 118, 509

Hartkopf, W. I., McAlister, H. A., Yang, X., \& Fekel, F. C. 1992, AJ, 103, 1976

Høg, E., Fabricius, C., Makarov, V. V., et al. 2000a, A\&A, 357, 367

Høg, E., Fabricius, C., Makarov, V. V., et al. 2000b, A\&A, 355, L27

Jancart, S., Jorissen, A., Babusiaux, C., \& Pourbaix, D. 2005, A\&A, 442, 365

Jorissen, A. 2003, in Asymptotic Giant Branch Stars, ed. H. Habing, \& H. Olofsson (New York: Springer Verlag), 461

Jorissen, A., \& Mayor, M. 1988, A\&A, 198, 187

Jorissen, A., \& Van Eck, S. 2005, in Cosmic Abundances as Records of Stellar

Evolution and Nucleosynthesis, ASP Conf. Ser., San Francisco, 336, 207

Jorissen, A., Van Eck, S., Mayor, M., \& Udry, S. 1998, A\&A, 332, 877 
Jorissen, A., Jancart, S., \& Pourbaix, D. 2004, in Spectroscopically and Spatially Resolving the Components of Close Binary Stars, ed. R. W. Hilditch, H. Hensberge, \& K. Pavlovski, ASP Conf. Ser., San Francisco, 318, 141

Jorissen, A., Začs, L., Udry, S., Lindgren, H., \& Musaev, F. A. 2005, A\&A, 441, 1135

Kaplan, G. H., \& Makarov, V. V. 2003, Astron. Nachr., 324, 419

Kim, C.-H., Lee, J. W., Kim, H.-I., Kyung, J.-M., \& Koch, R. H. 2003, AJ, 126, 1555

Knapp, G. R., Young, K., \& Crosas, M. 1999, A\&A, 346, 175

Knapp, G. R., Pourbaix, D., Platais, I., \& Jorissen, A. 2003, A\&A, 403, 993

Kouwenhoven, M. B. N., Brown, A. G. A., Zinnecker, H., Kaper, L., \& Portegies Zwart, S. F. 2005, A\&A, 430, 137

Latham, D. W., Stefanik, R. P., Torres, G., et al. 2002, AJ, 124, 1144

Levato, H., Malaroda, S., Morrell, N., \& Solivella, G. 1987, ApJS, 64, 487

Lü, P. K., Dawson, D. W., Upgren, A. R., \& Weis, E. W. 1983, ApJS, 52, 169

Makarov, V. V. 2004, ApJ, 600, L71

Makarov, V. V., \& Kaplan, G. H. 2005, AJ, 129, 2420

Mason, B. D., Gies, D. R., Hartkopf, W. I., et al. 1998, AJ, 115, 821

Mason, B. D., Hartkopf, W. I., Holdenried, E. R., \& Rafferty, T. J. 2001, AJ, 121, 3224

Massey, P., \& Niemela, V. S. 1981, ApJ, 245, 195

Mayor, M., \& Mazeh, T. 1987, A\&A, 171, 157

Mazeh, T. 1990, AJ, 99, 675

Mazeh, T., \& Shaham, J. 1979, A\&A, 77, 145

McClure, R. D. 1983, ApJ, 268, 264

McClure, R. D., Fletcher, J. M., \& Nemec, J. M. 1980, ApJ, 238, L35

McClure, R. D., \& Woodsworth, A. W. 1990, ApJ, 352, 709

Moffat, A. F. J., \& Niemela, V. S. 1982, A\&A, 108, 326

Nakajima, T., Oppenheimer, B. R., Kulkarni, S. R., et al. 1995, Nature, 378, 463 Niemela, V. S., Gamen, R., Morrell, N. I., \& Giménez Benítez, S. 1999, in Wolf-Rayet Phenomena in Massive Stars and Starburst Galaxies, ed. K. A. van der Hucht, G. Koenigsberger, \& P. R. J. Eenens (San Francisco: ASP), IAU Symp., 193, 26
Nordström, B., Mayor, M., Andersen, J., et al. 2004, A\&A, 418, 989 Patience, J., White, R. J., Ghez, A. M., et al. 2002, ApJ, 581, 654 Platais, I., Pourbaix, D., Jorissen, A., et al. 2003, A\&A, 397, 997

Pourbaix, D. 2004, in Spectroscopically and Spatially Resolving the Components of Close Binary Stars, ed. R. W. Hilditch, H. Hensberge, \& K. Pavlovski, ASP Conf. Ser., San Francisco, 318, 132

Pourbaix, D., \& Jorissen, A. 2000, A\&AS, 145, 161

Pourbaix, D., \& Boffin, H. M. J. 2003, A\&A, 398, 1163

Pourbaix, D., Platais, I., Detournay, S., et al. 2003, A\&A, 399, 1167

Pourbaix, D., Tokovinin, A. A., Batten, A. H., et al. 2004, A\&A, 424, 727

Proust, D., Ochsenbein, F., \& Pettersen, B. R. 1981, A\&AS, 44, 179

Renson, P., Gerbaldi, M., \& Catalano, F. A. 1991, A\&AS, 89, 429

Ribas, I., Arenou, F., \& Guinan, E. F. 2002, AJ, 123, 2033

Šleivyte, J., \& Bartkevičius, A. 1990, Vilnius Astron. Observ. Bull., 85, 3

Söderhjelm, S. 1999, A\&A, 341, 121

Tokovinin, A. A. 1997, A\&AS, 124, 75

Tokovinin, A. A. 2000, A\&A, 360, 997

Udry, S., Jorissen, A., Mayor, M., \& Van Eck, S. 1998, A\&AS, 131, 25

Udry, S., Mayor, M., Andersen, J., et al. 1997, in Hipparcos - Venice '97, ed. M. Perryman (Noordwijk: ESA), ESA SP-402, 693

Udry, S., Queloz, D., \& Mayor, M. 2003, in Scientific Frontiers in Research on Extrasolar Planets, ed. D. Deming, \& S. Seager, ASP Conf. Ser., San Francisco, 294, 17

Van Eck, S., Jorissen, A., Udry, S., Mayor, M., \& Pernier, B. 1998, A\&A, 329, 971

van Leeuwen, F., \& Evans, D. W. 1998, A\&AS, 130, 157

Vogt, S. S., Butler, R. P., Marcy, G. W., et al. 2002, ApJ, 568, 352

Wielen, R. 1997, A\&A, 325, 367

Wielen, R., Dettbarn, C., Jahreiß, H., Lenhardt, H., \& Schwan, H. 1999, A\&A, 346, 675

Wielen, R., Schwan, H., Dettbarn, C., Jahreiss, H., \& Lenhardt, H. 1998, Acta Historica Astronomiae, 3, 123 